\title{
ON ABELIAN EXTENSIONS OF SIMPLE RINGS
}

\author{
By
}

\section{Kazuo KISHIMOTO*)}

In [3], the author gave characterizations for a simple ring to have a cyclic extension, which generalizes the case of division ring extension treated by S. A. Amitsur in [1], while in [4], he studied as a generalized notion of a simple ring extension having a sigle commutative generator, a polynomial extension ${ }^{1)}$ of a simple ring as well as relationships between a cyclic extension and a Galois extension which is polynomial extension.

The present paper is a continuation of these earlier works to give, as main results, some necessary and sufficient conditions for a simple ring to have an abelian extension. $\$ \S 1-2$ are prerequisite preparations for the main purpose. In $\S 1$, we shall deal with an $e$-polynomial simple ring extension which is gneralized notion of a polynomial simple ring extension considered in. [4]. It can be seen from the definition that the structure of each $p^{e}$ dimensional fundamental abelian extension of prime characteristic $p$ and Kummer extension given in [Theorems 3,4 of [8] and Corollary 2 of [5]] are $e$-polynomial simple ring extensions. In $\$ 2$, we shall construct two types of noncommutative) polynomial ring with several indeterminates over a simple ring. One is defined by means of derivations and the other is defined by means of endomorphisms both in the basic simple ring. Further, as residue class ring of above polynomial rings, two types of $e$-polynomial simple ring extensions will be defined, the former of which is concerned with $p^{e}$-dimensional fundamental abelian extension of prime characteristic $p$ and the latter is concerned with a Kummer extension respectively.

Applying the results of the preceding sections, we shall give in $\S 3$, a necessary and sufficient condition for that a simple ring of prime characteristic $p$ have a $p^{e}$-dimensional fundamental abelian extension in Theorem 3.1, and also a necessary and sufficient condition for a $p^{f}$-dimensional abelian extension $T$ over $S$ of prime characteristic $p$ to be regularly embedded in a $p^{e}$-dimensional abelian extension $R / S$ such that $R / T$ is a fundamental abelian extension. Finally, $\S 4$ is devoted to the Kummer extension case. Namely, we shall obtain a necessary and sufficient condition for a simple ring to have an $n$-dimensional

*) This work was done while the author was a Domestic fellow in the Mathematical Institute, Hokkaido University, on leave from Hokkaido University of Education.

1) Cf. [2]. 
abelian extension (whose center contains a primitive $n$-th root $\zeta$ of 1 ) in Theorem 4.1. Moreover, in Theorem 4.2, a necessary and sufficient condition for that a $q$-dimensional abelian extension $T$ over $S$ such that the center of $T$ contains $\zeta$ and $T$ is $T_{l} S_{r}$-irreducible can be regularly embedded in a $q n$ dimensional abelian extension $R / S$ such that the center of $R$ contains $\zeta$ will be given.

Throughout the present paper, we use the following conventions:

Let $A$ be a ring and $a$ an element of $A$.

$A^{\circ}$ : the multiplicative group consisting of the regular elements of $A$.

$\tilde{A}:$ the set of all inner automorphisms determined by elements of $A^{\bullet}$.

$I_{a}, \tilde{a}$ : the inner derivation $a_{l}-a_{r}$ determined by $a$, the inner automorphism $a_{l} a_{r}^{-1}$ determined by the regular element $a$ respectively.

$J(\mathbb{S}, A)$ : the fixed-subring of $A$ under $\mathbb{S}$ where $\mathbb{S}$ is an automorphism group of $A$. of $A$.

$I_{a}|B, \mathbb{S}| B$ : the restriction of $I_{a}$ to $B$, of $\&$ to $B$ where $B$ is a subset

(S) $(B):\{\sigma \in \mathbb{S} ; b \sigma=b$ for all $b \in B\}$.

$A \backslash B$ : the set theoretical complement of $B$ in $A$,

$L N_{m}(a ; \rho)\left(\right.$ resp. $\left.R N_{m}(a ; \rho)\right): a \rho^{m-1} \cdot a \rho^{m-2} \cdots a \rho \cdot a\left(\right.$ resp. $\left.a a \rho \cdot a \rho^{m-1} a \rho^{m-1}\right)$ where $\rho$ is a (ring) homomorphism of $A$, by $N_{m}(a ; \rho)$ we denote $\operatorname{LN}_{m}(a ; \rho)$ if it coincides with $R N_{m}(a ; \rho)$.

$T_{m}(a ; \rho): \sum_{i=0}^{m-1} a d^{i t}$.

$A[X ; \rho, D]$ : the ring of all polynomials $\left\{X^{i} a_{i} ; a_{i} \in A\right\}$ which the multiplication is defined by $a X=X(\mathrm{a} \rho)+a D$ for each $a \in A$ where $D$ is a $\rho$-derivation in $A$. In particular, we set $A[X ; D]=A[X ; 1, D], A[X ; \rho]=A[P ; \rho, 0]$ and $A[X]=A[X ; 1,0]$.

$S, Z:$ a simple artinian ring, its center.

$R, C:$ an extension simple artinian ring of $S$ with common identity, its center.

$V: V_{R}(S)$, the centralizer of $S$ in $R$.

$\oplus M_{i}$ : a direct sum of modules $M_{i}$.

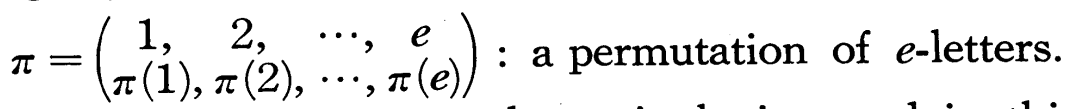

The other notations and terminologies used in this paper, we follow [3].

§ 1. e-polynomial simple ring extension.

First of all, we shall begin our study with $e$-polynomial simple ring extensions over $S$.

$R$ is called an $n$-dimensional right e-polynomial simple ring extension over $S$ if $R$ satisfies the following conditions: 
(1) $[R: S]_{r}=n$,

(2) $R=\sum^{0 \leq_{i} \leqq m_{j}-1} y_{e}^{\nu} y_{e-1}^{\nu} e_{-1}^{1} \cdots y_{1}^{\nu} S$ where $\prod_{i=1}^{e} m_{i}=n, m_{i}>1$,

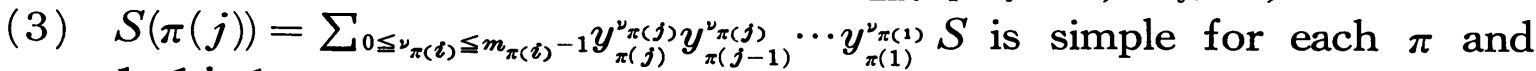
$1 \leqq j \leqq e$,

(4) $s y_{i}=y_{i} s_{1}^{(i)}+s_{0}^{(i)}\left(s_{0}^{(i)}, s_{1}^{(i)} \in S\right)$ for every $s \in S, y_{i} y_{j}=y_{j} y_{i} s_{i j}+t_{i j}$ for some $s_{i j}, t_{i j} \in S$.

(5) $y_{i}^{m_{i}}+\sum_{j=0}^{m_{i}-1} y_{i}^{j} u_{j}^{(i)}=0\left(u_{j}^{(i)} \in S\right)$.

Then, by $s \rightarrow s_{1}^{(i)}$, and $s \rightarrow s_{0}^{(i)}$, we can define a monomorphism $\rho_{i}$ in $S$ and $\rho_{i}$-derivation $D_{i}$ in $S$. Further, $s_{i j}$ is regular and $t_{i j}=-t_{j i} s_{i j}$ since $y_{i} y_{j}=$ $y_{j} y_{i} s_{i j}+t_{i j}=y_{i} y_{j} s_{j i} s_{i j}+t_{j i} s_{i j}+t_{i j}$. Moreover, by (3) and (4), we can see that each $\rho_{\pi(j)}$ can be extended a monomorphism $P_{\pi(j)}$ of $S(\pi(j))$ by $y_{\pi(i)} P_{\pi(j)}=$ $y_{\pi(i)} \boldsymbol{S}_{\pi(\xi) \pi(j)}$ and, a the same time, $D_{\pi(j)}$ can be extended a $P_{\pi(j)}$-derivation in $S(\pi(j))$.

An $n$-dimensional right $e$-polynomial simple ring extension $R$ over $S$ is called an n-dimensional e-polynomial simple ring extension over $S$ if $[R: S]_{l}$ $=n$. Throughout in this section, we assume that $R$ is an $n$-dimensional right $e$-polynomial simple ring extension over $S$, and in the rest, for the sake of simplicity, $\pi$ will be omitted if there is no danger of misunderstanding.

Lemma 1.1. $[S(j): S(i)]_{l}=\Pi_{p=i+1}^{j} \alpha_{p}$ for each $\pi$ and $i<j$ where $\alpha_{q}=$ $\sum_{j=1}^{m_{q}-1}\left([S(q): S(q) P]_{l}\right)^{j}+1$.

Proof. By (3) and (4), $S(j)$ is a right 1-polynomial simple ring extension over $S(j-1)(j=1,2, \cdots, e$ and we set $S(0)=S)$. Hence $[S(j): S(j-1)]_{l}=\alpha_{j}$ by [4. Theorem 1.1]. Thus the assertion is clear from the fact that $[S(j)$ : $S(i)]_{l}=[S(j): S(j-1)]_{l} .[S(j-1): S(j-2)]_{l} \cdots[S(i+1): S(i)]_{l}$.

Corollary 1.1. The following conditions are equivalent:

(a) $[R: S]_{\imath}=[R: S]_{r}$.

(b) $[S(j): S(i)]_{l}=[S(j): S(i)]_{r}$ for each $i<j$ and $i=0,1, \cdots, e-1$.

(c) each $\rho_{j}$ is an automorphism and it can be extended to an automorphism $P_{j}$ in $S(j)$ for ech $\pi$.

Proof. $(\mathbf{a}) \rightarrow(\mathbf{b})$ By Lemma 1.1, $[R: S]_{l}=\prod_{i=1}^{e} \alpha_{i}$ and $[S(i+1): S(i)]_{l} \geqq$ $[S(i+1): S(i)]_{r}$. Hence we obtain $(\mathbf{b})$.

$\left(\right.$ b) $\rightarrow\left(\right.$ c) Since $[S(1): S]_{l}=[S(1): S]_{r}, \rho_{1}$ is an automorphism in $S$ for each $\pi$. Next, $[S(j): S(j-1)]_{l}=[S(j): S(j-1)]_{r}$ shows that $P_{j}$ is an automorphism in $S(j)$.

$(\mathbf{c}) \rightarrow(\mathbf{a})$. This is a direct consquence of Lemma 1.1.

Corollary 1.2. If $D_{i}=0$ and $t_{i j}=0$ for each $i, j$, then $[R: S]_{r}=[R: S]_{l}$.

Proof. By [4. Corollary 1.2 (b)], $\rho_{i}=\widetilde{y}_{i}^{-1} \mid S$ is an automorphism in $S$. Further, $\left(y_{j}^{\nu, j} \cdot y_{j-1}^{\nu, j} \cdots y_{1}^{\nu} s\right) y_{i}=y_{i}\left(y_{j}^{\nu j} \cdot y_{j-1}^{\nu, j} \cdots y_{1}^{\nu} s\right) P_{i}$ shows that $P_{i}\left|S(j)=\widetilde{y}_{i}^{-1}\right| S(j)$. 
Hence we obtain $P_{i} \mid S(j)$ is an automorphism in $S(j)$. Thus the assertion is an immediate consequence of Corollary 1.1.

Proposition 1. 1. (a) If each $\rho_{i}=1$, and $s_{i j}=1$ then, $\left[D_{i}, D_{j}\right]=D_{i} D_{j}-D_{j} D_{i}=I_{i j}$ and $t_{i j} D_{k}+t_{j k} D_{i}+t_{k i} D_{j}=0$.

(b) If each $D_{i}=0$ and $t_{i j}=0$ then,

$$
\begin{aligned}
& \rho_{j}^{-1} \rho_{i}^{-1} \rho_{j} \rho_{i}=\widetilde{s}_{i j} \quad \text { and } \\
& s_{i j}\left(s_{i k} \rho_{j}\right) s_{j k}=\left(s_{j k} \rho_{i}\right) s_{i k}\left(s_{i j} \rho_{k}\right) .
\end{aligned}
$$

Proof. (a) Since $\rho_{i}=1$, we have $D_{i}=I_{y_{i}} \mid S$. Then, $s\left(D_{i} D_{j}-D_{j} D_{i}\right)=$ $s\left(y_{i} y_{j}-y_{j} y_{i}\right)-\left(y_{i} y_{j}-y_{j} y_{i}\right) s=s t_{i j}-t_{i j} s(s \in S)$ by the latter half condition of (4).

Next, $t_{i j} D_{k}+t_{j k} D_{i}+t_{k i} D_{j}=\left[\left[y_{i}, y_{j}\right], y_{k}\right]+\left[\left[y_{j}, y_{k}\right], y_{i}\right]+\left[\left[y_{k}, y_{i}\right], y_{j}\right]=0$.

(b) Since $D_{i}=0$, we may assume that $\rho_{i}=\widetilde{y}_{i}^{-1} \mid S$ is an automorphism in $S$ [4. Corollary 1.2]. Further, $y_{i} y_{j}=y_{j} y_{i} s_{i j}$ implies $s_{i j}=\left(y_{j} y_{i}\right)^{-1} y_{i} y_{j}$. Hence ${ }_{s} \rho_{j}^{-1} \rho_{i}^{-1} \rho_{j} \rho_{i}=y_{i}^{-1} y_{j}^{-1} y_{i} y_{j} s y_{j}^{-1} y_{i}^{-1} y_{j} y_{i}$ shows that $\rho_{j}^{-1} \rho_{i}^{-1} \rho_{j} \rho_{i}=\widetilde{s}_{i j}$.

Next, $\left(y_{i} y_{j}\right) y_{k}=y_{k}\left(y_{i} s_{i k} y_{j} s_{j k}\right)=y_{k}\left(y_{i} y_{j}\left(s_{i k} \rho_{j}\right) s_{j k}\right)=y_{k}\left(y_{j} y_{i}\left(s_{i j}\left(s_{i k} \rho_{j}\right) s_{j k}\right)\right.$. On the other hand, $\left(y_{i} y_{j}\right) y_{k}=\left(y_{j} y_{i} s_{i j}\right) y_{k}=y_{k}\left(y_{j} s_{j k} y_{i} s_{i k}\left(s_{i j} \rho_{k}\right)\right)=y_{k}\left(y_{j} y_{i}\left(s_{j k} \rho_{i}\right) \cdot s_{i k}\left(s_{i j} \rho_{k}\right)\right)$

Consequently, we have $s_{i j}\left(s_{s k} \rho_{j}\right) s_{j k}=\left(s_{j k} \rho_{i}\right) s_{i k}\left(s_{i j} \rho_{k}\right)$.

Proposition 1.2. Let $D_{i}=0, t_{i j}=0$ and $y_{i}^{m} i \in S$ for each $i$. Then $R$ is $R_{l} S_{r}$-irreducible if and only if $V$ is a division ring.

Proof. Let $V$ be a division ring. We show that $R=R x S$ for each $x \in R \backslash S$. Let $r=\sum y_{e}^{\nu} y_{e}^{\nu} e-1{ }^{1} \cdots y_{1}^{\nu_{1}} s_{\nu_{e} \cdots \nu_{1}}\left(s_{\nu} e_{\nu_{1}} \in S\right)$ be the shortest non zero relation relative to the representation by $\left\{y_{e}^{\nu} y_{e}^{\nu} e_{-1} \cdots y_{1}^{\nu 1} ; 0 \leqq \nu_{i} \leqq m_{i}-1\right\}$ in the element of $R x S$. Then we may assume that $r \in R x S \backslash S$ (otherwise, $r \in S$ implies $R=$ $R r S=R x S)$. Noting here $s r=\sum y_{e}^{\nu} y_{e}^{\nu} e_{-1}^{1} \cdots y_{1}^{\nu} s \rho_{e}^{\nu} e^{\nu} \ldots \rho_{1}^{\nu 1} s_{\nu_{e} \cdots \nu_{1}}(s \in S)$ is contained in $R x S$, we may set $r=y_{e}^{\alpha_{e}} y_{e-1}^{\alpha} e_{-1} \cdots y_{1}^{\alpha_{1}}+\sum y_{e}^{\mu} y_{e-1}^{\mu} e_{-1} \cdots y_{1}^{\mu_{1}} s_{\mu_{e} \cdots \mu_{1}}$ since $S \rho_{e}^{\alpha} \rho_{e}^{\alpha_{e}} e_{-1} \cdots \rho_{1}^{\alpha_{1}} S S$ $=S s S=S$ for each $s(\neq 0) \in S$. Then $\left(y_{e}^{\alpha_{e}} y_{e-1}^{\alpha_{e}}{ }^{1} \cdots y_{1}^{\alpha_{1}}\right)^{-1} r=1+\sum y_{e}^{u_{e}-\alpha_{e}} y_{e}^{\mu} e_{-1}{ }^{-\alpha_{e-1}} \cdots$ $y_{1}^{\mu_{1}-\alpha_{1}} t_{\mu_{e-} \alpha_{r} \cdots \mu_{1}-\alpha_{1}} \in R x S\left(t_{\mu_{e-}{ }^{\alpha} e^{\cdots} \mu_{1}-\alpha_{1}} \in S\right)$. Since $y_{i}^{m_{i}} \in S$, we may assume that $r=1$

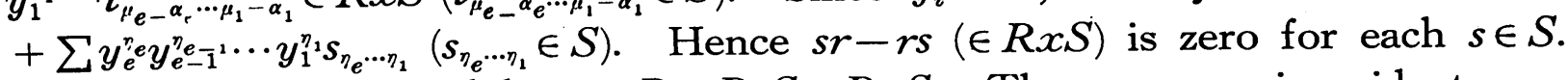
Consequently, $r \in V$ and hence $R=R r S=R x S$. The converse is evident.

Let $A$ be a simple artinian ring over $S$ such that $[A: S]_{r}$ is finite. $A / S$ is called (S)-regular if $A / S$ is Galois and there exists a Galois group (S) such that $\#(S)=[A: S]_{r}$, while $A$ is called an abelian extension over $S$ if $A / S$ is (S)-regular for some abelian $F$-group (S).

Theorem 1.1. Let $R$ be an n-dimensional right e-polynomial simple ring extension over $S$. Assume $n=p^{e}$, the characteristic $\chi(S)$ of $S$ is either zero or $>p, \rho_{i}=1$ and $s_{i j}=1$ for each $i, j$. Then,

(a) $V=Z$ implies $\mathbb{S B}(R / S)=1$ where $\mathbb{S S}(R / S)$ is the group of $S$-automor- 
phisms in $R$.

(b) $R / S$ is (S)-regular for some $\mathbb{B S}$ if and only if $R=S[C]$ and $C / Z$ is a pe-dimensional Galois extension.

Proof. (a) Since $\left(s y_{i}\right) \sigma=s\left(y_{i} \sigma\right)$ for each $\sigma \in \mathbb{S}(R / S)$ and $s \in S, y_{i} \sigma-y \in V$ $=Z$. Hence $y_{i} \sigma=y_{i}+s_{i \sigma}, s_{i \sigma} \in Z$. Thus we may assume that $y_{i} \sigma=y_{i}+1$ (e.g. take $\left.y_{i} s_{i o}^{-1}\right)$. If $y_{i}^{p}=\sum_{j=0}^{p-\mathfrak{I}} y_{i}^{j} s_{j}^{(i)}\left(s_{j}^{(i)} \in S\right)$, then $\left(y_{i}+1\right)^{p}=\sum_{k=0}^{p}\left(\begin{array}{l}p \\ k\end{array}\right) y_{i}^{k}=y_{i}^{p}+\sum_{k=0}^{p-1}$ $\left(\begin{array}{l}p \\ k\end{array}\right) y_{i}^{k}=\sum_{\substack{a-1 \\ k=0}} y\left(\left(\begin{array}{l}p \\ k\end{array}\right)+s_{k}^{(i)}\right)=y_{i}^{p} \sigma=\left(\sum_{j=0}^{p-1} y_{i}^{j} s_{j}^{(i)}\right) \sigma=\sum_{j=0}^{p-1}\left(\sum_{k=0}^{j}\left(\begin{array}{l}j \\ k\end{array}\right) y_{i}^{k} s_{j}^{(i)} D_{i}^{j-k}\right)$. From those, we can see that $p+s_{p-1}^{(i)}=s_{p-1}^{(i)}$, where it follows a contradiction $p=0$.

(b) Let $R / S$ be $\mathbb{S}$-regular for some $\mathbb{S}$. If $\sigma(\neq 1) \in \mathbb{S}$, then as is shown in the proof of $(\mathbf{a}), y_{i} \sigma=y_{i}+v_{i \sigma}\left(v_{i \sigma}(\neq 0) \in V\right)$, and we may assume $y_{i} \sigma^{k} \neq y_{i}$ for each $0<k<p^{j}$ where $p^{j}$ is the order of $\sigma$. Then $y_{i} \sigma^{p j}=y_{i}+T_{p j}\left(v_{i \sigma} ; \sigma\right)=y_{i}$ shows that $\#(\mathbb{S} \mid V)=p^{e}$ and hence $V=C$. Thus $R=S \otimes_{z} C$ and $J(\mathbb{S} \mid C, C)$ $=Z$ shows that $C / Z$ is a $p^{e}$-dimensional Galois extension. The converse is evident.

The following is a slight generalization of Jacobson's Theorem.

Theorem 1.2. Let $R$ be an n-dimensional right e-polynomial simple ring over $S$. Assume $n=2^{e}, \chi(S) \neq 2$ and $t_{i j}=0$ for each $i, j$. If $[S: Z]$ is finite, the $R / S$ is weakly Galois with respect to an abelian group (S) such that $\# \mathbb{B}=2^{e}$. In particular, if $R$ is a division ring, then $R / S$ is abelian.

Proof. Since $S(1) / S$ is a right quadratic extension for each $\pi$, we may assume that $y_{1}^{2} \in S$, and $S(1) / S$ is a Galois extension with respect to $\tau_{1}$ such that $y_{1} s \tau_{1}=-y_{1} s$ by [4. Corollary 4.1]. Let $\sigma_{i}$ be an extension of $\tau_{i}$ such that $y_{i} s \sigma_{i}=y_{i} s \tau_{i}$ and $y_{j} s \sigma_{i}=y_{j} s$ if $i \neq j$. Then $\sigma_{i}$ is an automorphism in $R$ since $t_{i j}=0$, and $J\left(\sigma_{i}, R\right)=S\left[y_{1}, \cdots, \dot{y}_{i}, \cdots, y_{e}\right]$. If we set $\mathbb{S}$, the group generated by $\sigma_{1}, \sigma_{2}, \cdots, \sigma_{e}$, then $\mathbb{S}=\left(\sigma_{1}\right) \times\left(\sigma_{2}\right) \times \cdots \times\left(\sigma_{e}\right)$ and $J(\mathbb{S}, R)=S$. If $R$ is a division ring, then so is $V$, and hence the above fact shows that $R / S$ is an abelian extension with respect to $\mathbb{B}$.

\section{$\S 2$. Construction of polynomial rings, e-polynomial simple ring extension.}

Let $\left(t_{i j}\right)$ be an $e \times e$ matrix with entries in $S$ such that $t_{i j}=-t_{j i}, t_{i i}=0$ and let $D_{1}, D_{2}, \cdots, D_{e}$ be derivations in $S$ satisfying $\left[D_{i}, D_{j}\right]=I_{t_{i j}}$ and $t_{i j} D_{k}$ $+t_{j k} D_{i}+t_{k i} D_{j}=0$. Further, by $S\left[\mathfrak{X}_{\pi(e)}\right]$ we denote $\left\{\sum X_{e}^{\nu_{e}} X_{e}^{\nu} X_{-1}{ }^{\nu} \cdots X_{1}^{\nu_{1}} s_{v_{e} \cdots \nu_{1}} ; s_{v_{e} \cdots \nu_{1}}\right.$ $\in S\}$, the set of all polynomials with $e$-indeterminates and the coefficients in $S$ (the coefficients written on the right hand and $X_{i}$ means $X_{\pi(i)}$ ). We define in $S\left[\mathfrak{X}_{\pi(e)}\right]$ the following multiplication rule and functions $E_{j}, j=1,2, \cdots, e$. 
(1) $s X_{e}^{\nu_{e}} X_{e-\overline{1}^{1}}^{\nu_{e}} \cdots X_{1}^{\nu_{1}}=\sum_{\alpha_{e}=0}^{\nu_{1}} \sum_{\alpha_{e-1}=0}^{\nu_{e-1}} \ldots \sum_{\alpha_{1}=0}^{\nu_{1}}\left(\begin{array}{c}\nu_{e} \\ \alpha_{e}\end{array}\right) \cdots\left(\begin{array}{c}\nu_{1} \\ \alpha_{1}\end{array}\right) X_{e}^{\nu_{e}-\alpha_{e} X_{e}^{\nu_{e} \overline{1}^{1}}{ }^{-\alpha_{e-1}} \ldots}$ $X_{1}^{\nu_{1}-\alpha_{1}} s D_{e}^{\alpha} e D_{e-1}^{\alpha}{ }^{1} \cdots D_{1}^{\alpha_{1}}$,

(2) $\left(X_{i}^{\nu} s\right) E_{j}=t_{i j} X_{i}^{\nu i^{-1}} s+X_{i} t_{i j} X_{i}^{{ }^{\nu} i^{-2}} s+X_{i}^{2} t_{i j} X_{i}^{\nu t^{-3}} s+\cdots+X_{i}^{\nu i^{-1}} t_{i j} s+X_{i}^{\nu}$ $\left(s D_{j}\right)$,

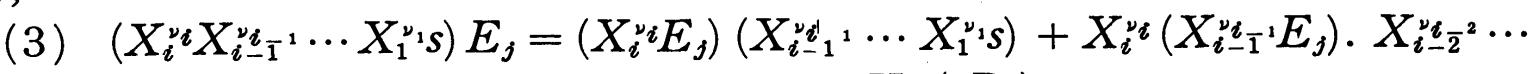
$X_{1}^{\nu_{1}} s+\cdots+X_{i}^{\nu_{i}} X_{i}^{\nu_{i}} \overline{-1}_{1} \cdots X_{2}^{\nu_{2}}\left(X_{1}^{\nu_{1}} E_{j}\right) s+X_{i}^{\nu} X_{i-1}^{\nu_{i}} \cdots X_{1}^{\nu_{1}}\left(s D_{j}\right)$,

(4) $\left(\sum_{k} Y_{k}\right) E_{j}=\sum_{k}\left(Y_{k} E_{j}\right)$ where $Y_{k}=X_{i}^{\nu_{i}} X_{i-1}^{\nu_{i}-1} \cdots X_{1}^{\nu_{1}} s_{\nu_{e} \cdots \nu_{1}}$ for each $s$, $s_{v e \ldots \nu_{1}} \in S$ and $i \leqq j$.

Proposition 2. 1. $S\left[\mathfrak{X}_{\pi(e)}\right]$ forms an associative ring by the following rule:

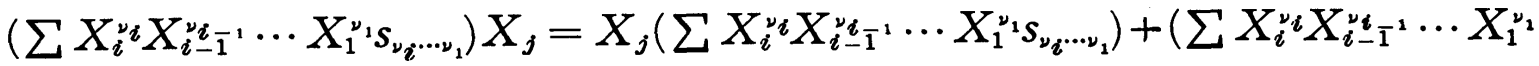
$\left.s_{\nu_{i}, \ldots \nu_{1}}\right) E_{j}$ for evch $j \geqq i$.

Moreover, when this is the case, $S\left[\mathfrak{X}_{\pi(e)}\right]$ is isomorphic to $S\left[\mathfrak{X}_{\pi^{\prime}(e)}\right]$ for every $\pi^{\prime}$.

Proof. We prove the assertion by induction on the number of indeterminates. $\left\{D_{1}, t_{11}=0\right\}$ satisfies the conditions stated above and $S\left[X_{1}, D_{1}\right]$ is an associative ring for each $\pi$. Hence we assume that $S\left[\mathfrak{X}_{\pi(e-1)}\right]=\left\{\sum X_{e-\overline{1}^{1}}\right.$ $\left.\cdots X_{1}^{\nu_{1}} s_{\nu_{e-1} \cdots \nu_{1}} ; s_{\nu_{e-1} \cdots \nu_{1}} \in S\right\}$ is an associative ring for each $\pi$. Then it is clear that the map $\sum X_{\pi(e-1)}^{\nu e-1} \cdots X_{\pi(1)}^{\nu_{1}} s_{\nu_{e-1} \cdots \nu_{1}} \rightarrow \sum X_{\pi^{\prime}\left(i_{e-1}\right)}^{\nu_{e-1}} \cdots X_{\pi^{\prime}\left(i_{1}\right)}^{\nu_{1}} s_{\nu_{e-1} \cdots \nu_{1}}$ gives an isomorphism of $S\left[\mathfrak{X}_{\pi(e-1)}\right]$ to $S\left[\mathfrak{X}_{\pi^{\prime}(e-1)}\right]$ where $\pi(j)=\pi\left(i_{j}\right)$. Now, the assertion is clear if we prove that $E_{e}$ is a derivation in $S\left[\mathfrak{X}_{\pi(e-1)}\right]$, namely, $f E_{e} X_{j}^{\nu}+f \cdot\left(X_{j}^{\nu} E_{e}\right)$ $=\left(\sum_{\alpha=0}^{\nu}\left(\begin{array}{c}\nu \\ \alpha\end{array}\right) X_{j}^{\nu-\alpha} f E_{j}^{\alpha}\right) E_{e}$ for each $f \in S\left[\mathfrak{X}_{\pi(j-1)}\right]$ and $j=1,2, \cdots, e-1$ (we set $\left.S\left[\mathfrak{X}_{\pi(0)}\right]=S\right)$ since $f X_{j}^{\nu}=\sum_{\alpha=0}^{\nu}\left(\begin{array}{c}\nu \\ \alpha\end{array}\right) X_{j}^{\nu-\alpha} f E_{j}^{\alpha} . \quad$ In $S\left[\mathfrak{X}_{\pi(e-1)}\right], X_{i} X_{j}=X_{j} X_{i}+t_{i j}=$ $X_{j} X_{i}-t_{j i}$ for each $j \geqq i$, and so we may assume that $E_{i}=I_{x_{i}} \mid S\left[\mathfrak{X}_{\pi(e-1)}\right], E_{j}=$ $I_{x_{j}} \mid S\left[\mathfrak{X}_{\pi(e-1)}\right]$ are derivations in $S\left[\mathfrak{X}_{\pi(e-1)}\right]$.

Firstly, we shall prove that $g\left[E_{j}, E_{k}\right]=g t_{j k}-t_{j k} g$ for each $g \in S\left[\mathfrak{X}_{\pi(e-1)}\right]$ and $j, k \leqq e-1$ by induction on the degree of $g$.

$X_{i} s\left[E_{j}, E_{k}\right]=X_{i}\left[E_{j}, E_{k}\right] s+X_{i}\left(s\left[E_{j}, E_{k}\right]\right)=\left(t_{i j} D_{k}-t_{i k} D_{j}\right) s+X_{i}\left(s t_{j k}-t_{j k} s\right)=$ $-t_{j k} D_{i} s+X_{i}\left(s t_{j k}-t_{j k} s\right)(s \in S)$ by the latter condition on $\left\{t_{i j}, D_{k}\right\}$. On the other hand, $X_{i} s t_{j k}-t_{j k} X_{i} s=X_{i}\left(s t_{j k}-t_{j k} s\right)-t_{j k} D_{i} s$. Therefore, we have $X_{i} s\left[E_{j}\right.$, $\left.E_{k}\right]=X_{i} s t_{j k}-t_{j k} X_{i} s . \quad$ Suppose that $\left(\prod_{\alpha=e-1}^{1} X_{\alpha}^{\nu} s\right)\left[E_{j}, E_{k}\right]=\left(\prod_{\alpha=e-1}{ }^{1} X_{\alpha}^{\nu} s\right) t_{j k}-t_{j k}\left(\prod_{\alpha=e-1}^{1}\right.$ $\left.X_{\alpha}^{\nu_{\alpha}} s\right)$ for each $\left(\prod_{\alpha=e-1}^{1} X_{\alpha}^{\nu_{\alpha}} s\right)(s \in S)$ such that $\sum_{\nu_{\alpha}}^{\nu}=m$. Let $\sum_{\alpha=1}^{\alpha=-1} \mu_{\alpha}=m+1$. Then $\left(\right.$ I $\left.^{1} X_{\alpha}^{\mu_{\alpha} s}\right)\left[E_{j}, E_{k}^{\alpha=e-1}=\left(X_{e}^{\mu_{e} e_{-}} \cdots X_{1}^{\mu_{1}-1}\left[E_{j}, E_{k}\right]\right) X_{1} s+\left(X_{e-1}^{\mu_{e}-1} \cdots X_{1}^{\mu_{1}-1}\right) \cdot\left(X_{1} s\left[E_{j}, E_{k}\right]\right)=\right.$

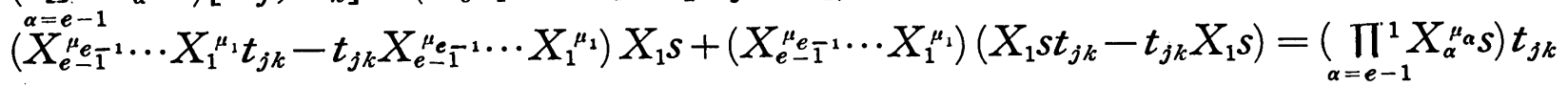
$-t_{j k}\left(\prod_{\alpha=e-1}^{1} X_{\alpha}^{\mu_{\alpha}} s\right)$. 
Next, let $f \in S\left[X_{\pi(j-1)}\right]$. Then $f E_{e} X_{j}+f \cdot\left(X_{j} E_{e}\right)-\left(X_{j} E_{e} f+X_{j} f E_{e}+f E_{j} E_{e}\right)$ $=X_{j} f E_{e}+f E_{e} E_{j}+f t_{j e}-\left(t_{j e} f+X_{j} f E_{e}+f E_{j} E_{e}\right)=f\left[E_{e}, E_{j}\right]-f t_{e j}+t_{e j} f$. Since we may assume that $f$ is contained in $S\left[\mathfrak{X}_{\pi^{\prime}(e-1)}\right]$ which contains $S\left[\mathfrak{X}_{\pi(j-1)}\right]$ and $X_{e}$ for a suitable $\pi^{\prime}, f\left[E_{e}, E_{j}\right]=f t_{e j}-t_{e j} f$. Thus we have $f E_{e} X_{j}+f X_{j} E_{e}=$ $\left(X_{j} f+f E_{j}\right) E_{e}=\left(f X_{j}\right) E_{e}$.

Next, we shall show that $\left(X_{j}^{q} f X_{j}\right) E_{e}=\left(X_{j}^{q} f E_{e}\right) X_{j}+\left(X_{j}^{q} f\right)\left(X_{j} E_{e}\right)$ for each $f \in S\left[\mathfrak{X}_{\pi(j-1)}\right] \cdots \cdots \cdots(\alpha)$.

$$
\begin{aligned}
\text { For, } & \left(X_{j}^{q} f X_{j}\right) E_{e}=\left(X_{j}^{q+1} f+X_{j}^{q} f E_{j}\right) E_{e} \\
& =X_{j}^{q} E_{e} X_{j} f+X_{j}^{q}\left(X_{j} f E_{e}\right)+X_{j}^{q} E_{e} f E_{j}+X_{j}^{q} f E_{j} E_{e} \\
& =X_{j}^{q} E_{e}\left(X_{j} f+f E_{j}\right)+X_{j}^{q}\left(X_{j} f E_{e}+f E_{j} E_{e}\right) \\
& =X_{j}^{q} E_{e}\left(f X_{j}\right)+X_{j}^{q}\left(f X_{j} E_{e}\right)=X_{j}^{q} E_{e}\left(f X_{j}\right)+X_{j}^{q}\left(f E_{e} X_{j}+f X_{j} E_{e}\right) \\
& =\left(X_{j}^{q} E_{e} f+X_{j}^{q} f E_{e}\right) X_{j}+X_{j}^{q} f X_{j} E_{e}=\left(X_{j}^{q} f\right) E_{e} X_{j}+\left(X_{j}^{q} f\right)\left(X_{j} E_{e}\right) .
\end{aligned}
$$

Let us assume that $\left(f X_{j}^{\nu-1}\right) E_{e}=f E_{e} X_{j}^{\nu-1}+f X_{j}^{\nu-1} E_{e}$. Then we can prove $\left(f X_{j}^{\nu}\right) E_{e}=f E_{e} X_{j}^{a}+f X_{j}^{\nu} E_{e}$ by use of $(\alpha)$.

For, $\left.\left(f X_{j}^{\nu}\right) E_{e}=\sum_{\mu=0}^{\nu-1}\left(\begin{array}{c}\nu-1 \\ \mu\end{array}\right) X_{j}^{\nu-\mu-1} f E_{j} X_{j}\right) E_{e}=\left(\sum_{\mu=0}^{\nu-1}\left(\begin{array}{c}\nu-1 \\ \mu\end{array}\right) X_{j}^{\nu-\mu-1} f E_{j}^{\mu}\right) E_{e} X_{j}$ $+\left(\sum_{\mu=0}^{\nu-1}\left(\begin{array}{c}\nu-1 \\ \mu\end{array}\right) X_{j}^{\nu-\rho-1} f E_{j}^{\mu}\right)\left(X_{j} E_{e}\right)=\left(f X_{j}^{\nu-1}\right) E_{e} X_{j}+\left(f X_{j}^{\nu-1}\right)\left(X_{j} E_{e}\right)$ $=f E_{e} X^{j}+f X_{j}^{\nu-1} E_{e} X_{j}+\left(f X_{j}^{\nu-1}\right)\left(X_{j} E_{e}\right)=f E_{e} X_{j}^{\nu}+f X_{j}^{\nu} E_{e}$.

Let $i=\pi^{\prime}\left(\alpha_{i}\right)$ for each $i=1,2, \cdots, e$. Then the map $\sum X_{e}^{\nu_{e}} X_{e-1}^{\nu_{e}-1^{1}} \cdots X_{1}^{\nu_{1}} s_{\nu_{e} \cdots \nu_{1}} \rightarrow$ $\sum X_{\pi^{\prime}\left(\alpha_{e}\right)}^{\nu_{e}} X_{\pi^{\prime}\left(\alpha_{e-1}\right)}^{\nu_{e}} \cdots X_{\pi^{\prime}(1)}^{\nu_{1}} \mathcal{S}_{\nu_{e} \cdots \nu_{1}}$ gives an isomorphim, of $S\left[\mathfrak{X}_{\pi(e)}\right]$ onto $S\left[\mathfrak{X}_{\pi^{\prime}(e)}\right]$.

By $S\left[\mathfrak{X}_{\pi(e)} ; \mathfrak{D}_{\pi(e)}\right]$ or sometimes, by $S\left[X_{1}, X_{2}, \cdots, X_{e} ; D_{1}, D_{2} \cdots, D_{e}\right]$ we denote the polynomials ring obtained in Proposition 2.1.

Let $\left(s_{i j}\right)$ be an $e \times e$ matrix with entries in $S^{\cdot}$ such that $s_{i j}=s_{j i}^{-1}, s_{i i}=1$, and let $\rho_{1}, \rho_{2}, \cdots, \rho_{e}$ be automorphisms in $S$ satisfying $\rho_{j}^{-1} \rho_{i}^{-1} \rho_{j} \rho_{i}=\tilde{s}_{i j}$ and $s_{i j}\left(s_{i k} \rho_{j}\right) s_{j k}=\left(s_{j k} \rho_{i}\right) s_{i k}\left(s_{i j} \rho_{k}\right)$. Further, we define the following multiplication rule and functions $P_{j}, j=1,2, \cdots, e$ in $S\left[\mathfrak{X}_{\pi(e)}\right]$.

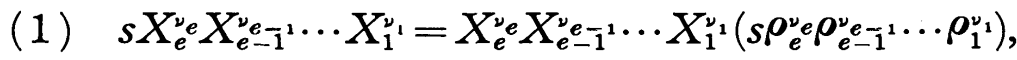

(2) $\left(X_{i}^{\nu_{i}} X_{i-1}^{\nu_{1}} \cdots X_{1}^{\nu_{1}} s\right) P_{j}=\left(X_{i} s_{i j}\right)^{\nu_{t}}\left(X_{i-1} s_{i-1 j}\right)^{\nu_{i}-1} \cdots\left(X_{1} s_{1 j}\right)^{\nu_{1}} s \rho_{j}$,

(3) $\left(\sum_{k} Y_{k}\right) P_{j}=\sum_{k}\left(Y_{k} P_{j}\right)$ where $Y_{k}=X_{i}^{\nu} X_{i-1}^{\nu} \cdots X_{1}^{\nu} s_{\nu_{2}}^{\nu} \cdots \nu_{1}$, for each $s, s_{\nu_{e} \ldots \nu_{1}} \in S$ and $j \geqq i$.

Proposition 2.2. $S\left[\mathfrak{X}_{\pi(e)}\right]$ forms an associative ring by the following rule:

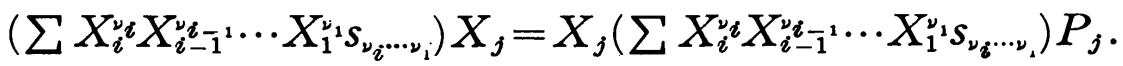

Moreover, when this is the case, $S\left[\mathfrak{X}_{\pi(e)}\right]$ is isomorphic to $S\left[\mathfrak{X}_{\pi^{\prime}(e)}\right]$ for each $\pi^{\prime}$.

Proof. We prove the assertion by induction on the number of indeterminates. $\quad\left\{\rho_{1}, s_{11}=1\right\}$ satisfies the conditions stated above and $S\left[X_{1} ; \rho_{1}\right]$ is an associative ring for each $\pi$. Hence we assume that $S\left[\mathfrak{X}_{\pi(e-1)}\right]$ is an associative 
ring for each $\pi$. Then it is clear that the map $\sum X_{\pi(e-1)}^{\nu} \cdots X_{\pi(1)}^{\nu_{1}} s_{\nu_{e-1} \cdots \nu_{1}} \rightarrow \sum X_{\pi^{\prime}\left(i_{e-1}\right)}^{\nu_{e}}$ $\cdots X_{\pi^{\prime}\left(i_{1}\right.}^{\nu_{1}} s_{\nu_{e-1} \cdots \nu_{1}}$ gives an isomorphism of $S\left[\mathfrak{x}_{\pi(e-1)}\right]$ to $S\left[\mathfrak{X}_{\pi^{\prime}(e-1)}\right]$ where $\pi(j)=$ $\pi^{\prime}\left(i_{j}\right)$. Now the assertion is clear if we prove that $P_{e}$ is a homomorphism in $S\left[\mathfrak{X}_{\pi(e-1)}\right]$, namely, $f P_{e} X_{j}^{\nu} P_{e}=X_{j}^{\nu} P_{e} f P_{j}^{\nu} P_{e}$ for each $f \in S\left[\mathfrak{X}_{\pi(j-1)}\right]$ and $j=1,2, \cdots, e-1$ (we set $S\left[\mathfrak{X}_{\pi(0)}\right]=S$ ) since $f \cdot X_{j}^{\nu}=X_{j}^{\nu} f P_{j}^{\nu}$. In $S\left[\mathfrak{X}_{\pi(e-1)}\right]$, each $P_{j}(j=1,2, \cdots, e-1$ ) is a homomorphism since $(f g) X_{j}=f\left(g X_{j}\right)$ for each $f, g \in S\left[\mathfrak{X}_{\pi(j-1)}\right]$. Then

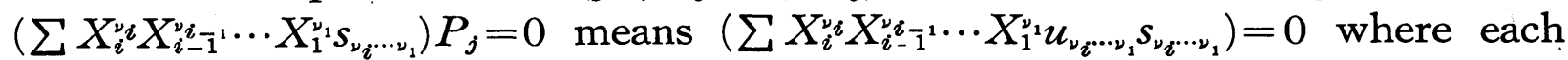
$u_{\nu_{i} \cdots \nu_{1}}$ is a regular element of $S$. Hence each $P_{j}$ is an automorphism in $S\left[\mathfrak{x}_{\pi(j)}\right]$. Firstly, we shall prove that $f P_{k}^{-1} P_{j}^{-1} \cdot P_{k} P_{j}=f \tilde{s}_{j k}$ for each $f \in S\left[\mathfrak{x}_{\pi(j-1)}\right]$ by induction on the degree of $f$.

For, $X_{i} s P_{k}^{-1} P_{j}^{-1} P_{k} P_{j}=X_{i} P_{k}^{-1} P_{j}^{-1} P_{k} P_{j} s \rho_{k}^{-1} \rho_{j}^{-1} \rho_{k} \rho_{j}$

$=X_{i} s_{i j}\left(s_{i k} \rho_{j}\right)\left(s_{j i} \rho_{j}^{-1} \rho_{k} \rho_{j}\right)\left(s_{k i} \rho_{k}^{-1} \rho_{j}^{-1} \rho_{k} \rho_{j}\right)\left(s \rho_{k}^{-1} \rho_{j}^{-1} \rho_{k} \rho_{j}\right)$

$\left.=X_{i}\left(s_{j k} \rho_{i} s_{i k}\right)\left(s_{i j} \rho_{k}\right) s_{k j}\right)\left(s_{j i} \rho_{j}^{-1} \rho_{k} \rho_{j}\right)\left(s_{j k} s_{k i} s_{k j}\right)\left(s_{\tilde{s}_{j k}}\right)$

$=X_{i}\left(s_{j k} \rho_{i} s_{i k}\left(s_{i j} \rho_{k}\right) s_{k j}\right) s_{j k}\left(s_{j i} \rho_{k}\right) s_{k j}\left(s_{j k} s_{k i} s_{k j}\right)\left(s \widetilde{s}_{j k}\right)$

$=X_{i}\left(s_{j k} \rho_{i} s_{k j}\right)\left(s \tilde{s}_{j k}\right)=\left(X_{i} \tilde{s}_{j k}\right)\left(s \tilde{s}_{j k}\right)$ by the latter conditions on $\left\{s_{i j}, P_{k}\right\}$.

Assume that $\left(X_{i}^{\nu_{i}} X_{i-1}^{\nu_{i}} \cdots X_{1}^{\nu} s\right) P_{k}^{-1} P_{j}^{-1} P_{k} P_{j}=\left(X_{i}^{\nu_{i}} X_{i-1}^{\nu_{i}} \cdots X_{1}^{\nu_{1}} s\right) \tilde{s}_{j k}$ for each $\sum_{j=1}^{i} \nu_{j}=m$. Let $\sum_{j=1}^{i} \mu_{j}=m+1$. Then $\left(X_{i}^{\mu_{i}} X_{i-1}^{\mu_{i}{ }^{1}} \cdots X_{1}^{\mu_{1}} s\right) P_{k}^{-1} P_{j}^{-1} P_{k} P_{j}=$ $\left(X_{i}^{\mu_{i}} X_{i-1}^{\mu_{i}} \cdots X_{1}^{\mu_{1}-1}\right) P_{k}^{-1} P_{j}^{-1} P_{k} P_{j}\left(X_{1} s\right) P_{k}^{-1} P_{j}^{-1} P_{k} P_{j}=\left(X_{i}^{\mu_{i}} X_{i-1}^{\mu_{i}{ }^{1}} \cdots X_{1}^{\mu_{1}-1}\right) \widetilde{s}_{j k}\left(X_{1} s\right) . \widetilde{\varepsilon}_{j k}$ $=\left(X_{i}^{\mu_{i}} X_{i-1}^{\mu_{i}} \cdots X_{1}^{\mu_{1}} s\right) \widetilde{s}_{j k}$.

Next, let $f \in S\left[\mathfrak{x}_{\pi(j-1)}\right]$. Then $f P_{e} X_{j} P_{e}-\left(X_{j} P_{e} f P_{j} P_{e}\right)=X_{j} . f P_{e} P_{j} s_{j e}-$ $X_{j} s_{j e} f P_{j} P_{e}=X_{j}\left(f P_{e} P_{j} s_{j e}-s_{j e} f P_{j} P_{e}\right)$. Since we may assume that $f P_{e}^{-1} P_{j}^{-1} P_{e} P_{j}$ $=f \widetilde{s}_{j e}$, we have $X_{j}\left(f P_{e} P_{j} s_{j e}-s_{j e} f P_{j} P_{e}\right)=0$. Suppose that $f P^{e} X_{j}^{\nu-1} P_{e}=$ $X_{j}^{\nu-1} P_{e} f P_{j}^{\nu-1} P_{j}^{j-1} P_{e}$. Then,

$f P_{e} X_{j}^{\nu} P_{e}=f P_{e} X_{j}^{\nu-1} P_{e} X_{j} P_{e}=X_{j}^{\nu-1} P_{e} f P_{j}^{\nu-1} P_{e} X_{j} s_{j e}=X_{j}^{a-1} P_{e} X_{j} f P_{j}^{\nu-1} P_{e} P_{j} s_{j e}$

$=X_{j}^{\nu-1} P_{e}\left(X_{j} P_{e} s_{e j}\right) f P_{j}^{\nu-1} P_{e} P_{j} s_{j e}=X_{j}^{\nu-1} P_{e} X_{j} P_{e} f P_{j}^{\nu-1} P_{e} P_{j} P_{j}^{-1} P_{e}^{-1} P_{j} P_{e}$

$=X_{j}^{\nu} P_{e} \cdot f P_{j}^{\nu} P_{e}$. If $i=\pi^{\prime}\left(\alpha_{i}\right)$ for each $i=1,2, \cdots, e$, the it is clear that $\sum X_{e}^{\nu} X_{e}^{v_{e}^{\prime} 1^{1}}$ $\cdots X_{1}^{\nu_{1}} s_{\nu_{e} \cdots \nu_{1}} \rightarrow \sum X_{\pi^{\prime}(e)}^{\nu_{e}} X_{\pi^{\prime}(e-1)}^{\left.\nu_{e}-1\right)} \cdots X_{\pi^{\prime}(1)}^{n_{1}} \boldsymbol{S}_{\nu^{\prime} e^{\cdots \nu_{1}}}$ gives an isomorphism of $S\left[\mathfrak{X}_{\pi(e)}\right]$ to $S\left[\mathfrak{X}_{\pi^{\prime}(e)}\right]$.

By $S\left[\mathfrak{X}_{\pi(e)} ; P_{\pi(e)}\right]$ or sometimes, by $S\left[X_{1}, X, \cdots, X_{e} ; \rho_{1}, \rho_{2}, \cdots, \rho_{e}\right]$, we denote the polynomials ring obtained in Proposition 2.2.

Lemma 2. 1. Let $f_{i}\left(X_{i}\right)=X_{i}^{m_{i}}+\sum_{j=0_{0}^{-1}}^{m_{i}} X_{i}^{j} s_{j}^{(i)}\left(s_{j}^{(i)} \in S\right)$ be a polynomial in $S\left[\mathfrak{x}_{\pi(e)}\right]$.

(a) If $f_{i}\left(X_{i}\right) \in S\left[\mathfrak{X}_{\pi(e)} ; D_{\pi(e)}\right]$, then $M=f_{i}\left(X_{i}\right) S\left[\mathfrak{X}_{\pi(e)} ; D_{\pi(i)}\right]$, a right ideal generated by $f_{i}\left(X_{i}\right)$ is a two-sided ideal if and only if $f_{i}\left(X_{i}\right)$ is central.

(b) If $f_{i}\left(X_{i}\right) \in S\left[\mathfrak{X}_{\pi(e)} ; P_{\pi(e)}\right]$, then $M$ is a two-sided ideal if and only if $\left\{s_{\nu}^{(i)}\right\}$ satisfies the following conditions:

(1) $s \boldsymbol{\rho}_{i}^{v} s_{\nu}^{(i)}=\boldsymbol{s}_{\nu}^{(i)} \boldsymbol{\rho}_{i}^{m_{i}}$

(2) $R N_{\nu}\left(s_{j i} ; \rho_{i}\right) s_{\nu}^{(i)}=s_{\nu}^{(i)} \rho_{j} R N_{m_{i}}\left(s_{j i} ; \rho_{i}\right)$. 
Proof. Since $f_{i}=f_{i}\left(X_{i}\right)$ is monic, $\operatorname{deg} f_{i} g=\operatorname{deg} f_{i}+\operatorname{deg} g$ for each polynomial $g$. Hence $M$ is a two-sided ideal if and only if $s f_{i}=f_{i} s^{\prime}\left(s^{\prime} \in S\right)$ for

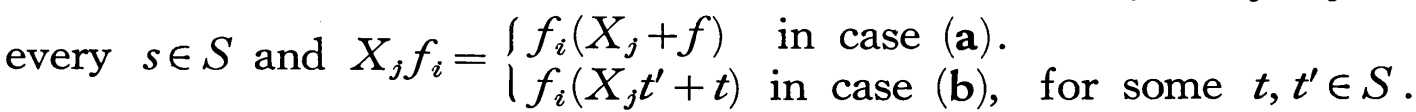

(a) Let $M$ be a two-sided ideal. Since $s f_{i}=X_{i}^{m_{i}} s+g_{i}\left(\operatorname{deg} g_{i}<m_{i}\right)$ and $f_{i} s^{\prime}=X_{i}^{m_{i}} s^{\prime}+g_{i}^{\prime}\left(\operatorname{deg} g^{\prime}<m\right)$, we can easily seen $s^{\prime}=s . \quad$ Next, $X_{j} f_{i}=f_{i} X_{j}+f_{i} E_{j}$ $=f_{i} X_{j}+f_{i} t$ show that $f_{i} E_{j}=f_{i} t$, and hence $t=0$ and $f_{i} E_{j}=0$. Thus $f_{i}$ is central. The converse is clear.

(b) $s f_{i}\left(X_{i}\right)=X_{i}^{m_{i}} s+\sum_{\nu=0}^{m_{i}-1} X_{i}^{\nu} s_{i}^{\nu} s_{\nu}^{(i)}$ and $f_{i}\left(X_{i}\right) s^{\prime}=X_{i}^{m_{i}} s^{\prime}+\sum_{\nu=0}^{m_{i}-1} X_{i}^{\nu} s_{\nu}^{(i)} s^{\prime}$, $=X^{j} f_{i}\left(X_{i}\right)=X_{i}^{m i}\left(X_{j} P_{i}^{m_{i}}\right)+\sum_{\nu=0}^{m_{i}-1} X_{i}^{\nu}\left(X_{j} P_{i}^{\nu}\right) s_{\nu}^{(i)}=X_{i}^{m_{i}} X_{j} R N_{m_{i}}\left(s_{j i} ; \rho_{i}\right)+\sum_{\nu=0}^{m_{i}-1}$

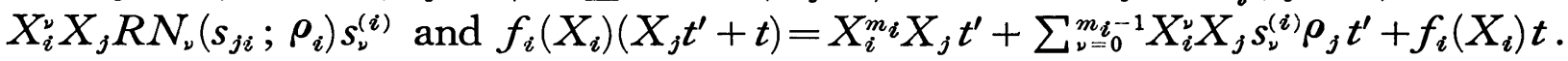
These facts show that $M$ is a two-sided ideal if and only if (1) and (2) are satisfied.

Lemma 2.2. Let $f_{i}\left(X_{i}\right)$ be a polynomial given in Lemma 2.1, $i=$ $1,2, \cdots, e$.

(a) If $f_{i}\left(X_{i}\right) \in S\left[\mathfrak{X}_{\pi(e)} ; \mathfrak{D}_{\pi(e)}\right]$ and $f_{i}\left(X_{i}\right)$ is central, then $M_{k} E_{j} \leqq M_{k}$ where $M_{k}=\left(f_{1}\left(X_{1}\right), f_{2}\left(X_{2}\right), \cdots, f_{k}\left(X_{k}\right)\right) S\left[\mathfrak{X}_{\pi(e)} ; \mathfrak{D}_{\pi(e)}\right]$.

(b) If $f_{i}\left(X_{i}\right)$ satisfies the conditions of Lemma 2.1 (b), then $M_{k} P_{j} \subseteq M_{k}$.

Proof. (a) Since $E_{j}=I_{X_{j}} \mid S\left[\mathfrak{X}_{\pi(e)} ; \mathfrak{D}_{\pi(e)}\right]$, the assertion is clear.

(b) $\quad\left(X_{i}^{m_{i}}+\sum_{\nu=0}^{m_{i}{ }^{-1}} X_{i}^{\nu} s_{\nu}^{(i)}\right) P_{j}=X_{i}^{m_{i}} L N_{m_{i}}\left(s_{i j} ; \rho_{i}\right)+\sum_{\nu=0}^{m_{i}-1} X_{i}^{\nu} L N_{\nu}\left(s_{i j} ; \rho_{i}\right) s_{\nu}^{(i)} . \quad$ If we note that $\left(L N_{\nu}\left(s_{i j}: \rho\right)\right)^{-1}=R N_{\nu}\left(s_{j i} ; \rho_{i}\right)$, the condition (2) of Lemma 2.1 (b) yields at once $M_{k} P_{j} \leqq M_{k}$.

By Lemma 2.2 , we can see that each $E_{j}\left(\right.$ resp. $\left.P_{j}\right)$ induces a derivation (resp. homomorphism) in $S\left[\mathfrak{X}_{\pi(k)} ; \mathfrak{D}_{\pi(k)}\right] / M_{k} \cong S\left[x_{1}, x_{2}, \cdots, x_{k}\right]$ (resp. $S\left[X_{\pi(k)}\right.$; $\left.\left.P_{\pi(k)}\right] / M_{k} \cong S\left[x_{1}, x_{2}, \cdots, x_{k}\right]\right)$ where $x_{i}$ is the residue class of $X_{i}$ modulo $M_{k}$. We denote by $E_{j}$ (resp. $P_{j}$ ), these induced derivation (resp. homomorphism) again. Then $S\left[x_{1}, x_{2}, \cdots, x_{k}\right]\left[X_{k+1} ; E_{k+1}\right]=\left\{\sum_{\nu} X_{k+1}^{\nu} f_{\nu} ; f_{\nu} \in S\left[x_{1}, x_{2}, \cdots, x_{k}\right]\right\}$ (resp. $\left.S\left[x_{1}, x_{2}, \cdots, x_{k}\right]\left[X_{k+1} ; P_{k+1}\right]\right)$ can be regarded as an associative ring by the rule $f X_{k+1}=X_{k+1} f+f E_{k+1}$ (resp. $\left.f X_{k+1}=X_{k+1} f P_{k+1}\right)$ ) for each $f \in S$ $\left[x_{1}, x_{2}, \cdots, x_{k}\right]$. Further, when this is the case, each polynomial $f_{k+1}\left(X_{k+1}\right)$ given in Lemma 2.1 can be considered as a polynomial in $S\left[x_{1}, x_{2}, \cdots, x_{k}\right]$ $\left[X_{k+1} ; E_{k+1}\right]\left(\operatorname{resp} . S\left[x_{1}, x_{2}, \cdots, x_{k}\right]\left[X_{k+1}: P_{k+1}\right]\right)$ and $f_{k+1}\left(X_{k+1}\right) S\left[x_{1}, x_{2}, \cdots, x_{k}\right]$ $\left[X_{k+1} ; E_{k+1}\right]$ is a two-sided ideal.

Now, let $S_{k}=S\left[\mathfrak{X}_{\pi(k)} ; \mathfrak{D}_{\pi(k)}\right] / f_{k}\left(X_{k}\right) S\left[\mathfrak{X}_{\pi(k)} ; \mathfrak{D}_{\pi(k)}\right] \cong S\left[w_{k}\right]\left[\mathfrak{X}_{\pi(k+1)} ; \mathfrak{D}_{\pi(k+1)}\right]$ where $w_{k}$ is the residue class of $X_{k}$ modulo $f_{k}\left(X_{k}\right) S\left[\mathfrak{X}_{\pi(k)} ; \mathfrak{D}_{\pi(k)}\right]$, and let $S_{i}=S_{i+1} / f_{i}\left(X_{i}\right) S_{i+1} \cong S\left[w_{k}, w_{k-1}, \cdots, w_{i}\right]\left[\mathfrak{X}_{\pi(i-1)} ; \mathfrak{D}_{\pi(i-1)}\right]$, (more precisely, $S\left[w_{k}\right]$ $\left[\mathfrak{X}_{\pi(k-1)} ; \mathfrak{D}_{\pi(k-1)}\right] / f_{k-1}\left(X_{k-1}\right) S\left[\mathfrak{w}_{k}\right]\left[\mathfrak{X}_{\pi(k-1)} ; \mathfrak{D}_{\pi(k-1)}\right] \cong S\left[\overline{\mathfrak{W}}_{k}, \mathfrak{w}_{k-1}\right]\left[\mathfrak{X}_{\pi(k-1)} ; \mathfrak{D}_{\pi(k-1)}\right]$ where $\overline{\mathfrak{r}}_{k}$ is the residue class of $\mathfrak{w}_{k}$ modulo $f_{k-1}\left(X_{k-1}\right) S\left[\mathfrak{w}_{k}\right]\left[\mathfrak{X}_{\pi\left(\pi_{-1}\right)} ; \mathfrak{D}_{\pi(k-1)}\right]$, but, by $w_{k}$ we denote $\bar{w}_{k}$ again). 
Lemma 2.3. (a) $S_{1}$ is isomorphic to $S\left[\mathfrak{X}_{\pi(k)} ; \mathfrak{D}_{\pi(k)}\right] / M_{k}$ (resp $S\left[\mathfrak{X}_{\pi(k)}\right.$; $\left.\left.\mathfrak{P}_{\pi(k)}\right] / M_{k}\right)$.

(b) If $f_{1}\left(X_{1}\right)$ is w-irreducible in $S_{2}$ for each $\pi$, then $S\left[x_{1}, x_{2}, \cdots, x_{k}\right]$ is simple for each $k$ and $\pi$.

Proof. The following diagram is commutative where $\varphi_{i}: S_{i+1} \rightarrow S_{i+1} /$ $f_{i}\left(X_{i}\right) S_{i+1}, \theta: S\left[\mathfrak{X}_{\pi(k)}: \mathfrak{D}_{\pi(k)}\right] \rightarrow S\left[\mathfrak{X}_{\pi(k)} ; \mathfrak{D}_{\pi(k)}\right] / M_{k}, \varphi=\varphi_{k} \varphi_{k-1} \cdots \varphi_{1}$, and $\Psi=($ an inverse image of $\varphi) \cdot \theta$.

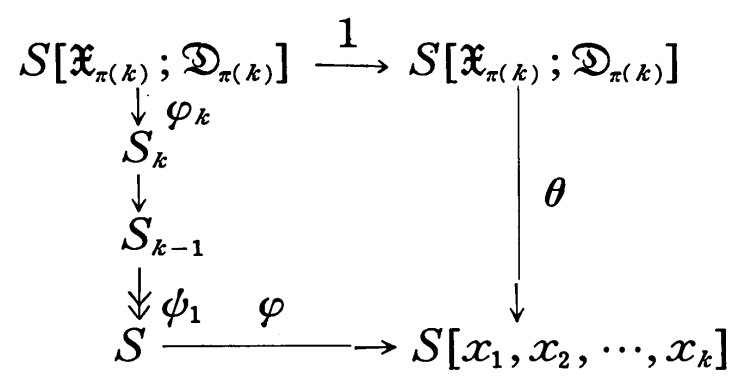

For, $S_{1} \ni \sum_{\nu} w_{k}^{\nu} w_{k-1}^{\nu} k_{-1}^{1} \cdots w_{1}^{\nu 1} S_{\nu_{k} \cdots \nu_{1}}=0(s \in S)$, then each inverse image of

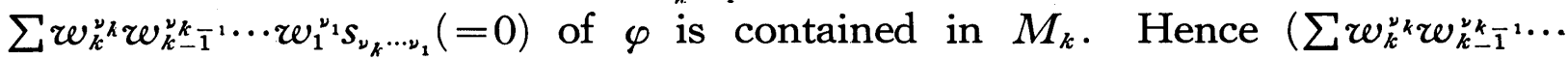
$\left.w_{1}^{\nu_{1}} S_{\nu_{k} w_{1}}\right) \Psi=0$, and this means that $\Psi$ is well defined. Next, let $\left(\sum w_{k}^{\nu_{k}{ }_{k}} w_{k-1}^{\nu_{k} k_{1}^{-1}}\right.$

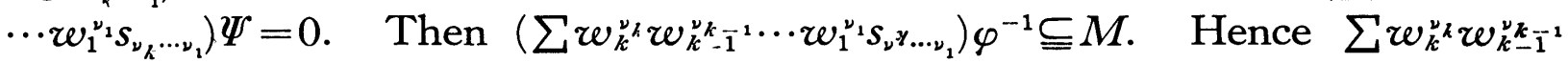
$\cdots w_{1}^{\nu_{1}} S_{\nu_{k} \cdots \nu_{1}}=0$.

(b) Since $S\left[w_{2}, w_{3}, \cdots, w_{e}\right] \supseteq S\left[w_{2}, w_{3}, \cdots, w_{k}\right] \supseteqq S$ for each $\pi$, the $w-$ irrducibility of $f_{1}\left(X_{1}\right)$ in $S_{e-1}$ yields at once that of $f_{1}\left(X_{1}\right)$ in $S\left[w_{2}, w_{3}, \cdots, w_{k}\right]$ $\left[X_{1} ; E_{1}\right]$. Hence $S\left[X_{1} ; D_{1}\right] / f_{1}\left(X_{1}\right) s\left[X_{1} ; D_{1}\right]$ is simple for each $\pi$. Thus repeating the procedures, we have the simplicity of $S\left[w_{1}, w_{2}, \cdots, w_{k}\right] \cong$ $S\left[x_{1}, x_{2}, \cdots, x_{k}\right]$.

Let $f_{i}\left(X_{i}\right)(i=1,2, \cdots, e)$ be a polynomial of $S\left[X_{i} ; D_{i}\right]$ (resp. $\left.S\left[X_{i} ; P_{i}\right]\right)$. Then $f_{i}\left(X_{i}\right)$ is called s.w-irreducible if $f_{i}\left(X_{i}\right)$ is w-irreducible in $S\left[X_{1}, X_{2}\right.$, $\left.\cdots, \breve{X}_{i}, \cdots, X_{k} ; E_{1}, E_{2}, \cdots, \breve{E}_{i}, \cdots, E_{k}\right] / M$ (resp. $S\left[X_{1}, X_{2}, \cdots, \breve{X}_{i}, \cdots, X_{k} ; P_{1}, P_{2}\right.$, $\left.\left.\cdots, \breve{P}_{i}, \cdots, P_{k}\right] / M\right)$ for each $1 \leqq k \leqq e$ where $M$ is a two-sided ideal generated by $f_{1}\left(X_{1}\right), f_{2}\left(X_{2}\right), \cdots, f_{i}\left(\check{X}_{i}\right), \cdots, f_{k}\left(X_{k}\right)$. Moreover, $f_{i}\left(X_{i}\right)$ is called s-irreducible if $f_{i}\left(X_{i}\right)$ is irreducible in $S\left[X_{1}, X_{2}, \cdots, \check{X}_{i}, \cdots, X_{k} ; E_{1}, E_{2}, \cdots, \breve{E}_{i}, \cdots, E_{k}\right] / M$ (resp. $S\left[X_{1}, X_{2}, \cdots, \check{X}_{i}, \cdots, X_{k} ; P_{1}, P_{2}, \cdots, \check{P}_{i}, \cdots, P_{k}\right] / M$ ).

In following we shall give necessary and sufficient conditions for a simple ring to have an $e$-polynomial simple ring extension of dimension $n=\prod_{i=1}^{e} m_{i}$, $m_{i}>1$.

Proposition 2.3. (a) In order that $S$ have an $n=\prod_{i=1}^{e} m_{i}(m>1)$-dimensional e-polynomial simple ring extension such that $\rho_{i}=1$ and $s_{i j}=1$, it is necessary and sufficient that there exist derivations $D_{1}, D_{2}, \cdots, D_{e}$ in $S,\left(t_{i j}\right)$ 
an $e \times e$ matrix with entries in $S$ such that $t_{i j}=-t_{j i}, t_{i i}=0$ and $\left(s_{\nu}^{(i)}\right) 1 \times m_{i}$ matrices $(i=1,2, \cdots, e)$ satisfying

(1) $\left[D_{i}, D_{j}\right]=I_{t_{i j}}$,

(2) $t_{i j} D_{k}+t_{j k} D_{i}+t_{k i} D_{j}=0$,

(3) $\quad\left(\begin{array}{c}m_{i}-k+h \\ h\end{array}\right) s D_{i}^{h} s_{m_{i-k+h}^{(i)}}^{(i)}=s_{m_{i}-k}^{(i)} s$,

(4) $s_{m_{i^{-k}}}^{(i)} D_{j}=\sum_{h=0}^{k}\left(\begin{array}{c}m_{i}-k+h \\ h\end{array}\right) t_{j i} D_{i}^{h-1} s_{m_{i^{-k+h}}^{(i)}}^{(5)}$

(5) $f_{i}\left(X_{i}\right)=X_{i}^{m_{i}}+\sum_{\nu=0}^{m_{i}-1} X_{i}^{\nu} s_{\nu}^{(i)}$ is s.w-irreducible.

(b) In order that $S$ have an $n=\prod_{i=1}^{e} m_{i}\left(m_{i}>1\right)$-dimensional e-polynomial simple ring extension such that $D_{i}=0$ and $t_{i j}=0$, it is necessary and sufficient that there exist automorphisms $\rho_{1}, \rho_{2}, \cdots, \rho_{e}$ in $S,\left(s_{i j}\right)$ an $e \times e$ matrix with entries in $S^{\bullet}$ such that $s_{i j}=s_{j i}^{-1}, s_{i i}=1$ and $\left(s_{\nu}^{(i)}\right) 1 \times m_{i}$ matrices $(i=1,2, \cdots, e)$ satisfying

(1) $\boldsymbol{\rho}_{j}^{-1} \boldsymbol{\rho}_{i}^{-1} \boldsymbol{\rho}_{j} \boldsymbol{\rho}_{i}=\widetilde{\varepsilon}_{i j}$,

(2) $s_{i j}\left(s_{i k} \rho_{j}\right) s_{j k}=\left(s_{j k} \rho_{i}\right) s_{i k}\left(s_{i j} \rho_{k}\right)$,

(3) $s \rho_{i}^{\nu} s_{\nu}^{(i)}=s_{\nu}^{(i)} s_{i}^{m_{i}}$,

(4) $R N_{\nu}\left(s_{j i} ; \rho_{j}\right) s_{\nu}^{(i)}=s_{\nu}^{(i)} \rho_{j} R N_{m_{i}}\left(s_{j i} ; \rho_{i}\right)$,

(5) $f_{i}\left(X_{i}\right)=X_{i}^{m_{i}}+\sum_{\nu=0}^{m_{i}-1} X_{i}^{\nu} s_{\nu}^{(i)}$ is s.w-irreducible.

Proof. By proposition 2.1, (1) and (2) are equivalent with the existence of a polynomial ring $S\left[\mathfrak{X}_{e} ; \mathfrak{D}_{e}\right]$. Let $M=\left(f_{1}\left(X_{1}\right), f_{2}\left(X_{2}\right), \cdots, f_{e}\left(X_{e}\right) S\left[\mathfrak{X}_{e} ; \mathfrak{D}_{e}\right]\right.$. Then (3) and (4) imply the fact that each $f_{i}\left(X_{i}\right)$ is central. Hence $M$ is a two-sided ideal. Now, by $(5), R^{*} \cong S\left[\mathfrak{X}_{e} ; \mathfrak{D}_{e}\right] / M$ is an $n=\prod_{i=1}^{e} m_{i}$-dimensional right $e$-polynomial simple ring extension over $S$. Further, if we note that each $\rho_{i}=1$ and $s_{i j}=1,\left[R^{*}: S\right]_{r}=\left[R^{*}: S\right]_{l}$ by Corollary 1.1. Conversely, let $R=S\left[x_{1}, x_{2}, \cdots, x_{e}\right]$ be an $n=\prod_{i=1}^{e} m_{i}\left(m_{i}>1\right)$-dimensional $e$-polynomial simple ring extension over $S$ such that $s x_{i}=x_{i} s+s_{0}^{(i)}\left(s_{0}^{(i)} \in S\right)$ for every $s \in S$ and $x_{i} x_{j}=x_{j} x_{i}+t_{i j}$. Then, by Proposition $1,1(\mathrm{a})$, each $D_{i}=I_{x_{i}} \mid S, t_{i j}=x_{i} x_{j}-x_{j} x_{i}$ satisfies (1) and (2). Let $x_{i}^{m_{i}}+\sum_{\nu=0}^{m_{i}-1} x_{i}^{\nu} s_{\nu}^{(i)}=0$. Then $s\left(x_{i}^{m_{i}}+\sum_{\nu=0}^{m_{i}-1} x_{i}^{\nu} s_{\nu}^{(i)}\right)=$ $\left(x_{i}^{m_{i}}+\sum_{\nu=0}^{m_{i}-1} x_{i}^{\nu} s_{\nu}^{(i)}\right) s$ and $x_{j}\left(x_{i}^{m_{i}}+\sum_{\nu=0}^{m_{i}{ }^{-1}} x_{i}^{\nu} s_{\nu}^{(i)}\right)=\left(x_{i}^{m_{i}}+\sum_{\nu \underline{0}_{0}^{-1}}^{m_{i}} x_{i}^{\nu} s_{\nu}^{(i)}\right) x_{j}$ imply (3) and (4) for each $1 \times m_{i}$ matrix $\left(s_{\nu}^{(i)}\right)$. Finally (5) is an immediate consequence of (3) of the definition of an e-polynomial simple ring extension and Lemma 2.3 .

(b) Sufficiency is clear from the proof of (a). Conversely, let $R=S$ $\left[x_{1}, x_{2}, \cdots, x_{e}\right]$ be an $n=\prod_{i=1}^{e} m_{i}\left(m_{i}>1\right)$-dimensional $e$-polynomial simple ring extension over $S$ with $s x_{i}=x_{i} s_{i}$ and $x_{i} x_{j}=x_{j} x_{i} s_{i j}$, then each $\tilde{x}_{i}^{-1} \mid S=\rho_{i}$, $x_{i}^{-1} x_{j}^{-1} x_{i} x_{j}=s_{i j}$ satisfies conditions (1) and (2). The rest of the proof is same 
as that of (a).

\section{§3. Abelian extensions of prime characteristic $p$.}

In this section, we shall deal with $p^{f}$-dimensional abelian extensions of a simple ring of prime characteristic $p$. A $p^{e}$-dimensional abelian extension $R$ over $S$ is called a $p^{e}$-dimensional fundamental abelian extension over $S$ if $\chi(S)=p$ and there exists a Galois gronp $\mathbb{S}=\left(\sigma_{1}\right) \times\left(\sigma_{2}\right) \times \cdots \times\left(\sigma_{e}\right)$, a direct product of $\sigma_{i}$, with $\# \sigma_{i}=p$ and $(S)$ is an $F$-group. Then, one of principal theorem of this section can be state

Theorem 3. 1. Let $\chi(S)=p$. In order that $S$ have a $p^{e}$-dimensional fundmaental abelian extension, it is necessary and sufficient that there exist derivations $D_{1}, D_{2}, \cdots, D_{e}$ in $S,\left(t_{i j}\right)$ an $e \times e$ matrix with entries in $S$ such that $t_{i j}=-t_{j i}, t_{i i}=0$ and $\left(s_{i}\right) 1 \times e$ matrix with entries in $S$ satisfying

(1) $\left[D_{i}, D_{j}\right]=I_{t_{i j}}$,

(2) $t_{i j} D_{k}+t_{j k} D_{i}+t_{k i} D_{j}=0$,

(3) $D_{i}^{p}-D_{i}=I_{s_{i}}, s_{i} D_{i}=0$,

(4) $t_{i j} D_{j}^{p-1}+t_{j i}+s_{j} D_{i}=0$

(5) $X_{\pi(i)}^{\mathfrak{p}}-s_{\pi(i)}=X_{\pi(i)}^{p}-X_{\pi(i)}-s_{\pi(i)}$ is s.w-irreducible.

More precisely, if there exists $\left\{D_{1}, D_{2}, \cdots, D_{e},\left(t_{i j}\right),\left(s_{i}\right)\right\}$ satisfying (1)-(5), then $M=\left(X_{1}^{\mathfrak{p}}-s_{1}, X_{2}^{\mathfrak{p}}-s_{2}, \cdots, X_{e}^{\mathfrak{p}}-s_{e}\right) S\left[\mathfrak{X}_{e} ; \mathfrak{D}_{e}\right]$ is a maximal ideal of $S\left[\mathfrak{X}_{e} ; \mathfrak{D}_{e}\right]$, $R^{*}=S\left[y_{1}, y_{2}, \cdots, y_{e}\right] \cong S\left[\mathfrak{X}_{e} ; \mathfrak{D}_{e}\right] / M$ is a $p^{e}$-dimensional fundamental abelian extension with respect to $\mathrm{SS}^{*}=\left(\sigma_{1}^{*}\right) \times\left(\sigma_{2}^{*}\right) \times \cdots \times\left(\sigma_{e}^{*}\right)$ such that $\# \sigma_{i}^{*}=p$ and $y_{i} \sigma_{j}^{*}=y_{i}+\delta_{i j}$ where each $y_{i}$ is the residue class of $X_{i}$ modulo $M$. Conversely, if $R$ is a pe-dimensional fundamental abelian extension over $S$ with respect to $\mathbb{S}=\left(\sigma_{1}\right) \times\left(\sigma_{2}\right) \times \cdots \times\left(\sigma_{e}\right)$, then we can find such $\left\{D_{1}, D_{2}, \cdots, D_{e},\left(t_{i j}\right),\left(s_{i}\right)\right\}$ satisfying (1)-(5) that there holds an S-isomorphism $\varphi^{*}: R^{*} \cong R$ with $\varphi^{*} \sigma_{i}$ $=\sigma_{i}^{*} \varphi^{*}$.

Proof. Since $s\left(X_{i}^{p}-X_{i}-s_{i}\right)=X_{i}^{p} s-X_{i} s+s D_{i}^{p}-s D_{i}-s s_{i}$, and $X_{j}\left(X_{i}^{p}-X_{i}\right.$ $\left.-s_{i}\right)=X_{i}^{p} X_{j}+X_{i} X_{j}-s X_{j}+s_{j i} D_{i}^{p-1}-s_{j i}+s_{i} D_{j}$, (3) and (4) are equivalent with $X_{i}^{\mathfrak{p}}-s_{i}$ is central in $S\left[\mathfrak{X}_{e} ; \mathfrak{D}_{e}\right]$. Thus, by Proposition 2.3 (a), $R^{*}=S\left[y_{1}, y_{2}\right.$, $\left.\cdots, y_{e}\right] \cong S\left[\mathfrak{X}_{e} ; \mathfrak{D}_{e}\right] / M$ is a $p^{e}$-dimensional $e$-polynomial simple ring extension over $S$. Let $\Phi_{i}$ be the map of $S\left[\mathfrak{X}_{e} ; \mathfrak{D}_{e}\right]$ defined by $\sum_{\nu} X_{i}^{\nu} f_{\nu} \rightarrow \sum_{\nu}\left(X_{i}+1\right)^{\nu} f_{\nu}\left(f_{\nu} \in\right.$ $\left.S\left[X_{1}, X_{2}, \cdots, \check{X}_{i}, \cdots, X_{e} ; D_{1}, D_{2}, \cdots, \check{D}_{i}, \cdots, D_{e}\right]\right)$. Then $\left(f \Phi_{i}\right) X_{i} \Phi_{i}=f^{\bullet}\left(X_{i}+1\right)$ $=X_{i} f+f E_{i}+f=\left(X_{i} f+f E_{i}\right) \Phi_{i}$ shows that $\Phi_{i}$ is a ring homomorphism of $S\left[\mathfrak{X}_{e}: \mathfrak{D}_{e}\right]$, and moreover, $\Phi_{i}^{p}=1$ (since $\chi(S)=p$ ) means that $\Phi_{i}$ is an automorphism of order $p$. From the definition of $\Phi_{i}, \Phi_{i} \Phi_{j}=\Phi_{j} \Phi_{i}$ is clear. Next, $\left(X_{i}^{\mathfrak{p}}-s_{i}\right) \Phi_{j}=\left(X_{i}+\delta_{i j}\right)^{p}-\left(X_{i}+\delta_{i j}\right)-s=X_{i}^{\mathfrak{p}}-s$. Hence each $\Phi_{j}$ induces an automorphism $\sigma_{j}^{*}$ of order $p$ in $R^{*}$. Let $\left(\sigma_{j}^{*}\right)_{\cap}\left(\sigma_{1}^{*}, \sigma_{2}^{*}, \cdots, \sigma_{j-1}^{*}\right) \ni \sigma^{*}=\sigma_{j}^{* k}$. Then 
$y_{j} \sigma^{*}=y_{j}+k=y_{j} \sigma_{1}^{* \alpha_{1}} \sigma_{2}^{* \alpha_{2} \cdots} \sigma_{j-1}^{* \alpha_{j-1}}=y_{j}$ show that $\sigma^{*}=1$. Consequently, we have (S) ${ }^{*}$, the group generated by $\sigma_{1}^{*}, \sigma_{2}^{*}, \cdots, \sigma_{e}^{*}$, is an abelian group of order $p$ such that the direct product of $\sigma_{1}^{*}, \sigma_{2}^{*}, \cdots, \sigma_{e}^{*}$. Noting here $J\left(\sigma_{i}^{*}, R^{*}\right)=S\left[y_{1}, y_{2}, \cdots\right.$, $\left.\check{y}_{i}, \cdots, y_{e}\right]$, we have $J\left(\mathbb{S}^{*}, R^{*}\right)=\bigcap_{i=1}^{e} J\left(\sigma_{i}^{*}, R^{*}\right)=S$. Thus $R^{*} / S$ is a $p^{e}$-dimensional weakly Galois extension with respect to (S)* Thus in following, we shall show that $V^{*}=V_{R^{*}}(S)$ is simple. By [Lemma 2 of [6]], it suffices to prove that $C^{*}\left[v_{1}, v_{2}, \cdots, v_{k}\right]$ is a field where $\left\{\widetilde{v}_{1}, \widetilde{v}_{2}, \cdots, \widetilde{v}_{k}\right\}=\mathbb{S}^{*}{ }_{n} \widetilde{\boldsymbol{V}}^{*}$.

Since $\widetilde{v}_{i} \sigma_{j}^{*}=\sigma_{j}^{*} \widetilde{v}_{i}, v_{i} \sigma_{j}^{*}=c_{i j} v_{i}$ for some $c_{i j} \in C^{*}=V^{R^{*}}\left(R^{*}\right)$ (and $\widetilde{v}_{i} \widetilde{v}_{j}=\widetilde{v}_{j} \widetilde{v}_{i}$ since $v_{i} \widetilde{v}_{j}=c \cdot v_{i}$ for some $c \in C^{*}$ implies $\left.v_{i}=v_{i} \widetilde{v}_{j}^{p}=c^{p} v_{i}\right)$. Noting here that $C^{*}$ is cyclic over $J\left(\sigma_{j}^{*} \mid C^{*}, C^{*}\right)$ and $N_{p}\left(c_{i j} ; \sigma_{j}^{*}\right)=1$, there exists an element $d_{i j} \in C^{*}$ such that $c_{i j}^{-1}=d_{i j}^{-1}\left(d_{i j} \sigma_{j}^{*}\right)$. Setting here $u_{i j}=v_{i} d_{i j}$, then $u_{i j}$ is contained in $V_{S\left[s_{1}, y_{2} \cdots \breve{y}_{j}, \cdots, y_{e}\right]}(S)=V_{j}^{*}$. Hence $v_{i} \in C^{*}\left[V_{j}^{*}\right]$ for erch $j=1,2, \cdots, e$, and so, $v_{i} \in \bigcap_{j=1}^{e} C^{*}\left[V_{j}^{*}\right]$. Noting that $\bigcap_{j=1}^{e} C^{*} \otimes_{C^{*} \cap S} V^{*}=C^{*} \otimes_{C^{*} \cap S}\left(\bigcap_{j=1}^{e} V_{j}^{*}\right)=C^{*}$. $\otimes_{C^{*} \cap S} Z$ (considered in $C^{*} \otimes_{C^{*} \cap S} V^{*}$ ) and $\bigcap_{j=1}^{e} C^{*}\left[V_{j}^{*}\right]$ is an epimorphic image of $\bigcap_{j=1}^{e} C^{*} \otimes_{C^{*} S_{S}} V_{j}^{*}$, we have $v_{i} \in C^{*}[Z]$. Now we shall show that $C^{*}[Z]$ is a field. For, each $z \in Z, z E_{k}=z y_{k}-y_{k} z \in Z$ and hence, $z^{p} E_{k}=p\left(z E_{k} z^{p-1}\right)=0$. Hence $z^{p} \in C^{*}{ }_{n} S$. While, $C^{*} \otimes_{C^{*} \otimes s} Z$ is a commutative semi-simple artinian ring since $C^{*} / C^{*}{ }_{n} S$ is a finite Galois extension field. Let $u v=0$ for some $u, v \in C^{*} \otimes_{C^{*} \cap s} Z$. Then $0=(u v)^{p}=u^{p} v^{p}$ yields at once $u^{p}=0$ or $V^{p}=0$ since $u^{p}, v^{p} \in C^{*}$, and hence $u=0$ or $v=0$. Thus $C^{*} \otimes_{C^{*} \cap s} Z \cong C^{*}[Z]$ is a field. Consequently, $C^{*}\left[v_{i}\right]$ is a field since $\left[C^{*}\left[v_{i}\right]: C^{*}\right]<\infty$. Repeating the same procedures, we have $C^{*}\left[v_{1}, v_{2}, \cdots, v_{k}\right] \cong\left(C^{*}[Z]\right)$ is a field.

Conversely, let $R$ be a $p^{e}$-dimensinal fundamental abelian extension over $S$ with respect to $\mathbb{B S}=\left(\sigma_{1}\right) \times\left(\sigma_{2}\right) \times \cdots \times\left(\sigma_{e}\right)$. Then $R=S\left[x_{1}, x_{2}, \cdots, x_{e}\right]$ with $x_{i} \sigma_{j}=x_{i}+\delta_{i j}$ [Corollary 2 of [5]]. Then $D_{i}=I_{x_{i}} \mid S$ is a derivation in $S$ since $\left(s x_{i}-x_{i} s\right) \sigma_{j}=s\left(x_{i}+\delta_{i j}\right)-\left(x_{i}+\delta_{i j}\right) s=s x_{i}-x_{i} s$. Further, $\left(x_{i} x_{j}-x_{j} x_{i}\right) \sigma_{k}=\left(x_{i}\right.$ $\left.+\delta_{i k}\right) . \quad\left(x_{j}+\delta_{j k}\right)-\left(x_{j}+\delta_{j k}\right)\left(x_{i}+\delta_{i k}\right)=x_{i} x_{j}-x_{j} x_{i} . \quad$ Hence, if we set $t_{i j}=x_{i} x_{j}$ $-x_{j} x_{i}$, then $t_{i j}=-t_{j i}, t_{i i}=0$ and $\left\{D_{1}, D_{2}, \cdots, D_{e},\left(t_{i j}\right)\right\}$ satisfies conditions (1) and (2). Thus there exists a polynomial ring $S\left[\mathfrak{X}_{e} ; \mathfrak{D}_{e}\right]$. Next, $\left(x_{i}^{p}-x_{i}\right) \sigma_{j}=$ $\left(x_{i}+\delta_{i j}\right)^{p}-\left(x_{i}+\delta_{i j}\right)$ shows that $s_{i}=x_{i}^{p}-x_{i} \in S$ and $X_{i}^{\mathfrak{p}}-s_{i}$ is central in $S\left[\mathfrak{X}_{e}\right.$; $\mathfrak{D}_{e}$ ]. Hence conditions (3) and (4) are satisfied. Finally, if we note that $V$ is a field [Theorem 5 of [5]], each intermediate ring of $R / S$ is simple [Lemma 1.4 of [7]]. Hence $X_{i}^{p}-s_{i}$ is $s . w$-irreducible. Let $\varphi^{*}$ be the map defined

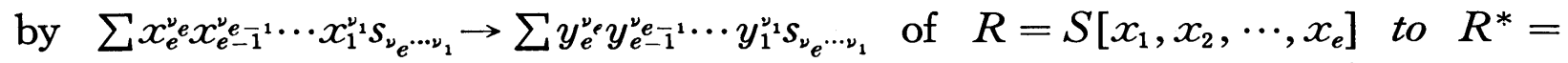
$S\left[y_{1}, y_{2}, \cdots, y_{e}\right]$, then it is clear that $\varphi^{*}$ is an $S$-isomrophism of $R$ to $R^{*}$ satisfying $\varphi^{*} \sigma_{i}^{*}=\sigma_{i} \varphi^{*}$. 
Corollary 3.1. Let $\chi(S)=p$. (a) In order that $S$ have a $p^{e}$-dimensional fundamental inner abelian extension, it is necessary and sufficient that there exist $\left\{D_{1}, D_{2}, \cdots, D_{e},\left(t_{i j}\right),\left(s_{i}\right)\right\}$ satisfying (1)-(4) of Theorem 3.1 and $\left(z_{i}\right)$ a $1 \times e$ matrix with entries in $Z^{*}$ satisfying

(6) $z_{i} D_{j}=z_{i} \delta_{i j}$.

(b) In order that $S$ have a $p^{e}$-dimensional fundamental outer abelian extension, it is necessary and sufficient that there exist $\left\{D_{1}, D_{2}, \cdots, D_{e},\left(t_{j i}\right)\right.$, $\left.\left(s_{i}\right)\right\}$ satisfying (1)-(5) of Theorem 3.1 and

$\left(6^{\prime}\right) \quad z D_{j}=0$ for all $j=1,2, \cdots, e$.

Proof. By Proposition 2.3 (a), there exists a $p^{e}$-dimensional $e$-polynomial simple ring extension $R^{*}=S\left[y_{1}, y_{2}, \cdots, y_{e}\right]$ over $S$ where each $y_{i}$ is the residue class of $X_{i}$ modulo $M$. Let $\sum_{\nu=0}^{p-1} X_{j}^{\nu} f_{\nu}$ be a polynomial of $S\left[y_{1}, y_{2}, \cdots, y_{j-1}\right]$ $\left[X_{j} ; E_{j}\right]\left(f_{\nu} \in S\left[y_{1}, y_{2}, \cdots, y_{j-1}\right]\right)$. Then, for each $z_{j}$ satisfying $(6), z_{j}\left(\sum_{\nu=0}^{p-1} X_{j}^{\nu} f_{\nu}\right)$ $=\left(\sum_{\nu=0}^{p-1} \sum_{i=0}^{\nu}\left(\begin{array}{l}\nu \\ i\end{array}\right) X_{j}^{i} z_{j} f_{\nu}\right)=\sum_{\nu=0}^{p-1}\left(\sum_{i=0}^{\nu}\left(\begin{array}{l}\nu \\ i\end{array}\right) X_{j}^{i} f_{\nu} z_{j}\right)$. Hence we have $\left(\sum_{\nu=0}^{p-1} X_{j}^{\nu} f_{\nu}\right)$ $I_{x_{j}}^{p}=(p-1) ! z_{j}^{p-1} f_{p-1}$ by induction on the degree of the polynomial. Thus each $X_{i}^{\mathfrak{p}}-s_{i}$ is s.w-irreducible. Consequently, $R^{*}$ is a $p^{e}$-dimensional fundamental abelian extension of $S$. Next, $z_{i} D_{j}=z_{i} y_{j}-y_{j} z_{i}=z_{i} \delta_{i j}$ shows that $z_{i} y_{j} z_{i}^{-1}=$ $y_{j}+\delta_{i j}$. Thus we may adapt $\tilde{z}_{i}$ as $\sigma_{i}^{*}$ since $z_{i}^{p} \in C^{*}$.

Conversely, let $R / S$ be a $p^{e}$-dimensional fundamental inner abelian extension over $S$ with respect to $\mathbb{S}=\left(\widetilde{v}_{1}\right) \times\left(\widetilde{v}_{2}\right) \times \cdots \times\left(\tilde{v}_{e}\right), v_{i} \in V$. Since $V=C[Z]$ [Theorem 5 of [5]], we have $v_{i}=\sum_{k} c_{k}^{(i)} z_{k}$ where $\left\{c_{k}^{(i)}\right\}(\subseteq C)$ is a $Z$-basis for $V$. On the other hand, $v_{i} x_{j} v_{i}^{-1}=x_{j}+\delta_{i j}$ implies $v_{i} E_{j}=v_{i} x_{j}-x_{j} v_{i}=v_{i} \delta_{i j}$. Hence $v_{i} E_{j}=\sum_{k} c_{k}^{(i)}\left(z_{k}^{(i)} E_{j}\right)=v \delta_{i j}$ shows that the existence of $\left(z_{1}, z_{2}, \cdots, z_{e}\right)$ satisfying (6).

(b) Under the same notations of the proof of (a), if (B)* contains $\tau=$ $\sigma_{1}^{* \alpha_{1}} \sigma_{1}^{* \alpha_{2}} \cdots \sigma_{k}^{* \alpha_{k}}=\widetilde{v}\left(v \in V^{*}\right)$, as was shown in the latter half of the proof of (a), there exists $z \in Z^{\bullet}$ such that $z D_{j}=\alpha_{j} z(j=1,2, \cdots, k)$, and this contradicts $Z D_{j}=0$ for all $j=1,2, \cdots, \mathrm{e}$.

Conversely, if $R$ is a $p^{e}$-dimensional outer Galois extension over $S$, then $Z \subseteq C$. Hence $Z D_{j}=0$ since $C D_{j}=0$ for all $j=1,2, \cdots, e$.

$R$ is called a trivial extension over $S$ if $R=S[C]$. Then we can prove the following:

Corollary 3.2. Let $\chi(S)=p$. Then the following conditions are equivalent.

(a) $S$ has a pe-dimensional fundamental trivial abelian extension.

(b) There exist elements $z_{1}, z_{2}, \cdots, z_{e}$ in $Z^{\cdot}$ such that the algbraic set associated with a two-sided ideol $M=\left(X_{1}^{\mathfrak{p}}-z_{1}, X_{2}^{\mathfrak{p}}-z, \cdots, X_{e}^{\mathfrak{p}}-z_{e}\right) Z\left[X_{1}, X_{2}\right.$, $\left.\cdots, X_{e}\right]$ is an algebraic variety.

(c) There exist elements $s_{1}, s_{2}, \cdots, s_{e}$ in $S$ such that $X_{i}^{\mathfrak{p}}-s_{i}$ is s.w-irre- 
ducible in $S\left[X_{1}, X_{2}, \cdots, X_{e}\right]$.

Proof. $\quad(\mathbf{a}) \rightarrow(\mathbf{b}) \quad$ Let $R=S[C]=S \otimes_{Z} C$. Then $J(\mathfrak{S} \mid C, C)=Z$ means that $C / Z$ is a $p^{e}$-dfmensional fundamental abelian extension with the (abelian) Galois group $(\mathfrak{S} \mid C$. Hence $(\mathrm{b})$ is clear.

$(\mathbf{a}) \rightarrow(\mathbf{c})$ Let $R=S[C]$ and $\left\{c_{e}^{\nu} e_{e}^{\nu} e_{-1}{ }^{1} \cdots c_{1}^{\nu_{1}} ; 0 \leqq \nu_{i} \leqq p-1, c_{i} \in C\right\}$ be $Z$-basis such that $c_{i}^{p}-c_{i} \in Z$. Then $X_{i}^{\mathfrak{p}}-c_{i}$ is $s \cdot w$-irreducible in $S\left[X_{1}, X_{2}, \cdots, X_{i}\right]$.

(b) $\rightarrow\left(\right.$ a) Since $Z^{\prime}=Z\left[X_{1}, X_{2}, \cdots, X_{e}\right] / M$ is a $p^{e}$-dimensional fundamental abelian extension, $S \otimes_{z} Z^{\prime}$ is a requested one.

$(\mathbf{c}) \rightarrow(\mathbf{a}) \quad$ Let $\quad M=\left(X_{1}^{\mathfrak{p}}-s_{1}, X_{2}^{\mathfrak{p}}-s_{2}, \cdots, X_{e}^{\mathfrak{p}}-s_{e}\right) S\left[X_{1}, X_{2}, \cdots, X_{e}\right] . \quad$ Then $S\left[X_{1}, X_{2}, \cdots, X_{e}\right] / M$ is a requested one.

Let $X_{i}^{\mathfrak{p}}-s_{i}$ be $s$-irreducible. Then $S\left[\mathfrak{X}_{e} ; \mathfrak{D}_{e}\right] / M$ is a division ring. Hence we obtain

Corollary 3.3. Let $S$ be a division ring of $\chi(S)=p$. In order that $S$ have a pe-dimensional fundamental abelian division ring extension, it is necessary and sufficient that there exist $\left\{D_{1}, D_{2}, \cdots, D_{e},\left(t_{i j}\right),\left(s_{i}\right)\right\}$ satisfying (1)-(4) of Theorem 3.1 and

(5') $X_{i}^{\mathfrak{p}}-s_{i}$ is s-irreducible.

Let $R / S$ be a $p^{e}$-dimensional fundamental abelian extension with respect to (S). Then $R / S$ is called a parallel extension if $R=S[T]$ such that $T / T_{\cap} S$ is a $p^{e}$-dimensional fundamental abelian extension with respect to $B \mid T$. A $p^{e}$-dimensional fundamental parallel abelian extension is called $a$ d-parallel (resp an $f$-parallel) extension if $T$ can be a division ring (resp. a field).

Corollary 3.4. Let $\chi(S)=p$. (a) In order that $S$ have a $p^{e}$-dimensional fundamental parallel extension, it is necessary and sufficient that there exist $\left\{D_{1}, D_{2}, \cdots, D_{e},\left(t_{i j}\right),\left(s_{i}\right)\right\}$ satisfying (1)-(5) of Theorem 3.1 and

(6) there exists a simple subring $W(\ni 1)$ of $S$ satisfying $W D_{i} \subseteq W$, $\left\{\left\{t_{i j}\right\},\left\{s_{i}\right\}\right\} \subseteq W$ and $X_{i}^{\mathfrak{p}}-s_{i}$ is $s \cdot w$-irreducible.

(b) In order that $S$ have a $p^{e}$-dimensional fundamental d-parallel abelian extension, it is necessary and sufficient that there exist $\left\{D_{1}, D_{2}, \cdots, D_{e},\left(t_{i j}\right)\right.$, $\left.\left(s_{i}\right)\right\}$ satisfying (1)-(5) of Theorem 3.1 and

$\left(6^{\prime}\right)$ there exists a divison subring $W(\ni 1)$ of $S$ satisfying $W D_{i} \subseteq W$, $\left\{\left\{t_{i j}\right\},\left\{s_{i}\right\}\right\} \subseteq W$ and $X_{i}^{\mathfrak{p}}-s_{i}$ is s-irreducible in $W\left[\mathfrak{X}_{\pi(e)} ; \mathfrak{D}_{\pi(e)}\right]$.

(c) In order that $S$ have a $p^{e}$-dimensional fundamental $f$-parallel abelian extension, it is necessary and sufficient that there exist $\left\{D_{1}, D_{2}, \cdots, D_{e},\left(t_{i j}\right)\right.$, $\left(s_{i}\right)\{$ satifying (1)-(5) of Theorem 3.1 and

$\left(6^{\prime \prime}\right)$ there exists a subfield $W(\ni 1)$ of $S$ satisfying $X_{i}^{\mathfrak{p}}-s_{i}$ is s-irreducible in $W\left[X_{1}, X_{2}, \cdots, X_{e}\right]$.

Proof. Let $R^{*}=S\left[y_{1}, y_{2}, \cdots, y_{e}\right] \cong S\left[\mathfrak{X}_{e} ; \mathfrak{D}_{e}\right] / M$, and $T^{*}=\left\{\sum y_{e}^{\nu} y_{e-1}^{\nu}{ }_{e}{ }^{1} \cdots\right.$ 
$\left.y_{1}^{\nu_{1}} w_{\nu_{e} \cdots \nu_{1}} ; w_{\nu_{e} \nu_{\nu_{1}}} \in W\right\}$. Then $J\left(S^{*} \mid T^{*}, T^{*}\right)=T^{*} S=W$ and $p^{e}=\# \mathbb{S S}^{*}=\left[T^{*}\right.$ : $W]$. and $R=S\left[T^{*}\right]$.

(a) Since $T^{*} \cong W\left[\mathfrak{X}_{e} ; \mathfrak{D}_{e}\right] / M_{W}, M_{W}=\left(X_{1}^{\mathfrak{p}}-s_{1}, X_{2}^{\mathfrak{p}}-s_{2}, \cdots, X_{e}^{\mathfrak{p}}-s_{e}\right) W\left[\mathfrak{X}_{e} ;\right.$ $\left.\mathfrak{D}_{e}\right], T^{*}$ is a simple ring.

Conversely, if $R=S[T]$ is a $p^{e}$-dimeesional fundamental parallel abelian extension with respect to $(S)$, then $T / T_{\cap} S$ is a $p^{e}$-dimensional fundamental abelian extension with respect to $\mathcal{S} \mid T$. Hence there exists $\left\{t_{1}, t_{2}, \cdots, t_{e}\right\} \subseteq T$ with $t_{i} \sigma_{j}=t_{i}+\delta_{i j}$. If we set $D_{i}=I_{t_{i}} \mid S, t_{i j}=t_{i} t_{j}-t_{j} t_{i}, s_{i}=t_{i}^{p}-t_{i}$ and $W=T_{n} S$, then our assertion is easy by the proof of Theorem 3.1.

(b) Combining (a) with Corollary 3.3, we readily obtain (b).

(c) Combining (a) with Corollary 3.2, we readily obtain (c).

Now, in the following, we shall deal with a regularly embedding problem of an abelian extension.

Let $R / S$ be (S)-regular, and an intermediate ring $T$ of $R / S$ be satisfy $J(\mathfrak{E}, T)=S$ and $[T: S]=\# \mathfrak{L}$ where $\mathfrak{S} \equiv \mathbb{S}$. If $\mathfrak{T}=\mathbb{S} \mid T, T / S$ is said to be regularly embedded in $R / S$.

Let $T / S$ be a $p^{e}$-dimensional abelian extension with respect to $\mathfrak{Z}=$ $\left(\tau_{1}\right) \times\left(\tau_{2}\right) \times \cdots \times\left(\tau_{m}\right)$, \#( $\left.\tau_{i}\right)=p^{f_{i}}$. We shall give a necessary and sufficient condition for $T / S$ can be regularly embedded in $R / S$ where $R / S$ is a $p^{f+m+k_{-}}$ dimensional abelian extension with respect to SS $=\left(\sigma_{1}\right) \times\left(\sigma_{2}\right) \times \cdots \times\left(\sigma_{m}\right) \times\left(\sigma_{m+1}\right)$ $\times \cdots \times\left(\sigma_{m+k}\right)$ if $k \geqq 0$ and (S) $=\left(\sigma_{1}\right) \times\left(\sigma_{2}\right) \times \cdots \times\left(\sigma_{m+k}\right) \times\left(\tau_{m+k+1}\right) \times \cdots \times\left(\tau_{m}\right)$ if $-m$ $<k<0$.

Let $s$ be an element of $S \subseteq S\left[\mathfrak{X}_{e} ; \mathfrak{D}_{e}\right]$, and let $\Delta_{0}^{(i)}(s)=1$ and $\Delta_{k}^{(i)}(s)=\Delta_{k-1}^{(i)}(s) D_{i}$ $+\Delta_{k-1}^{(i)}(s) s$. Then we have $\left(X_{i}+s\right)^{n}=\sum_{k=0}^{n}\left(\begin{array}{l}k \\ n\end{array}\right) X_{i}^{k} \Delta_{n-k}^{(i)}(s)$ [§1. I, (ii) of [4]].

Theorem 3.2. Let $\chi(S)=p$ and $T / S$ be a $p^{f}$-dimensional abelian extension with respect to $\mathfrak{S}=\left(\tau_{1}\right) \times\left(\tau_{2}\right) \times \cdots \times\left(\tau_{m}\right)$, \# $\tau_{i}=p^{f_{i}} . \quad$ In order that $T / S$ can be regularly embedded to a $p_{f+m+k}$-dimensional abelian extension such that $k \geqq-m$, it is necessary and sufficient that there exist derivations $D_{1}, D_{2}, \cdots, D_{m}, \cdots, D_{m+k}$ in $T,\left(t_{i j}\right)$ an $(m+k) \times(m+k)$ matrix with entries in $T,\left(t_{i}\right)$ a $1 \times(m+k)$ matrix with entries in $T$ satisfying the conditions (1)-(5) of Theorem 3.1 (in $T$ ) and $\left(b_{i j}\right)$ an $(m+k) \times(m+k)$ matrix with entries in $T$ satisfying

(1) $D_{i} \tau_{j}-\tau_{j} D_{i}=\tau_{j} I_{b_{i j}}$,

(2) $T_{\# \tau_{j}}\left(b_{i j} ; \tau_{j}\right)=\delta_{i j}$,

(3) $\Delta_{p}^{(i)}\left(b_{i j}\right)-b_{i j}=t_{i}\left(\tau_{j}-1\right)$,

(4) $t_{i j}\left(\tau_{k}-1\right)=b_{i k}\left(\tau_{k}^{-1} D_{j} \tau_{k}\right)-b_{j k} D_{i}$,

(5) $b_{i j}\left(\tau_{k}-1\right)=b_{i k}\left(\tau_{j}-1\right)$

More precisely, if there exists $\left\{D_{1}, \cdots, D_{m+k},\left(t_{i j}\right),\left(t_{i}\right),\left(b_{i j}\right)\right\}$ satisfying the 
conditions, then $R^{*}=T\left[y_{1}, y_{2}, \cdots, y_{m}, y_{m+1}, \cdots, y_{m+k}\right]=T\left[\mathfrak{X}_{m+k} ; \mathfrak{D}_{m+k}\right] / M, M=$ $\left(X_{1}^{\mathfrak{p}}-t_{1}, X_{2}^{\mathfrak{p}}-t_{2}, \cdots, X_{m+k}^{\mathfrak{p}}-t_{m+k}\right) T\left[\mathfrak{X}_{m+k} ; \mathfrak{D}_{m+k}\right]$ and $y_{k}$ is the residue class of $X_{k}$ modulo $M$, is a requested one with generating automorphisms $\sigma_{1}^{*}, \sigma_{2}^{*}, \ldots$, $\sigma_{m}^{*}, \cdots, \sigma_{m+k}^{*}$ such that $\left(y_{i} t\right) \sigma_{j}^{*}=\left(y_{i}+b_{i j}\right)\left(t \tau_{j}\right)(t \in T)$ and $\mathbb{S B}^{*} \mid T=\mathfrak{S}$ where (SF $^{*}=$ $\left(\sigma_{1}^{*}\right) \times\left(\sigma_{2}^{*}\right) \times \cdots \times\left(\sigma_{m}^{*}\right) \times \cdots \times\left(\sigma_{m+k}^{*}\right)$ if $k \geqq 0$ and SS $^{*}=\left(\sigma_{1}^{*}\right) \times\left(\sigma_{2}^{*}\right) \times \cdots \times\left(\sigma_{m+k}^{*}\right) \times$ $\left(\tau_{m+k+1}\right) \times \cdots \times\left(\tau_{m}\right)$ if $k<0$. Conversely, if $T / S$ can be regularly embedded in a $p^{r+m+k}$-dimensional abelian extension with respect to $\mathbb{S}=\left(\sigma_{1}\right) \times\left(\sigma_{2}\right) \times \cdots \times\left(\sigma_{m+k}\right)$ then there exists such $\left\{D_{1}, D_{2}, \cdots, D_{m+k},\left(t_{i j}\right),\left(t_{i}\right),\left(b_{i j}\right)\right\}$ satisfying the conditions in Theorem 3.1 and (1)-(5) that there holds a T-isomorphism $\varphi^{*}: R^{*} \cong R$ with $\varphi^{*} \sigma_{i}=\sigma_{i}^{*} \varphi^{*}$.

Proof. Firstly, we set $b_{i j}=\delta_{i j}$ if $k>0, j>m$ or $b_{i j}=0$, if $k<0$ and $j>m$. Then, clearly, they satisfy the conditions (1)-(5).

Let $\Phi_{j}(j=1,2, \cdots, m+k)$ be the mapping of $T\left[\mathfrak{X}_{m+k} ; \mathfrak{D}_{m+k}\right]$ defined by

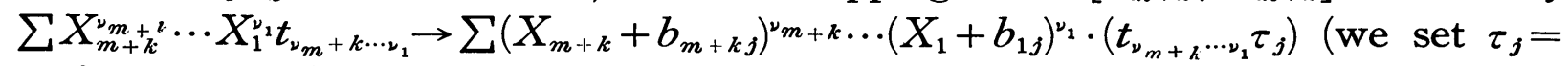
1 if $j>m)$. Then we can prove that $\Phi_{j}$ is an automorphism by induction on the number of inderteminates. By (1), we have $\left(t \Phi_{j}\right) X_{i} \Phi_{j}=\left(t \tau_{j}\right)\left(X_{i}+b_{i j}\right)=$ $X_{i}\left(t \tau_{j}\right)+t \tau_{j} D_{i}+t \tau_{j} b_{i j}=X_{i} t \tau_{j}+t D_{i} \tau_{j}-\left(t \tau_{j} b_{i j}-b_{i j} t \tau_{j}\right)+t \tau_{j} b_{i j}=X_{i} t \tau_{j}+t D_{i} \tau_{j}+$ $b_{i j} t \tau_{j}=\left(X_{i} t+t D_{i}\right) \Phi_{j}$. Hence $\Phi_{j}$ is a (ring) epimorphism of $T\left[X_{i} ; D_{i}\right]$. Further $\left(\sum_{\nu=0}^{m} X_{i}^{v} t_{\nu}\right) \Phi_{j}=\sum_{\nu=0}^{m}\left(X_{i}+b_{i j}\right)^{\nu}\left(t_{\nu} \tau_{j}\right)=X_{i}^{m} t_{m} \tau_{j}+X_{i}^{m-1}\left(\left(\begin{array}{c}m \\ m-1\end{array}\right) b_{i j} D_{i} t_{m} \tau_{j}+t_{m-1} \tau_{j}\right)+$ $\cdots+\left(\Delta_{m}^{(i)}\left(b_{i j}\right) t_{m} \tau_{j}+\cdots+t_{0} \tau_{j}\right)$ shows that $\Phi_{j}$ is an automorphism. Hence, we assume that $\Phi_{j}$ is an automorphism in $T\left[\mathfrak{X}_{m+k-1} ; \mathfrak{D}_{m+k-1}\right]$. Thus it suffices to prove $\left(Y \Phi_{j}\right)\left(X_{m+k} \Phi_{j}\right)=\left(X_{m+k} \Phi_{j}\right)\left(Y \Phi_{j}\right)+\left(Y E_{m+k}\right) \Phi_{j}$ where $Y \in T\left[\mathfrak{X}_{m+k-1}\right.$; $\left.\mathfrak{D}_{m+k-1}\right]$. Now, $\left(Y \Phi_{j}\right)\left(X_{m+k} \Phi_{j}\right)=\left(Y \Phi_{j}\right)\left(X_{m+k}+b_{m+k j}\right)=X_{m+k}\left(Y \Phi_{j}\right)+\left(Y \Phi_{j}\right) E_{m+k}$ $+\left(Y \Phi_{j}\right) b_{m+k j}$ and $\left(X_{m+k} \Phi_{j}\right)\left(Y \Phi_{j}\right)+\left(Y E_{m+k}\right) \Phi_{j}=\left(X_{m+k}+b_{m+k j}\right)\left(Y \Phi_{j}\right)+\left(Y E_{m+k}\right) \Phi_{j}$. Hence the assertion is clear if we show that $\left(Y \Phi_{j}\right) I_{b_{m+k} j}=Y\left(E_{m+k} \Phi_{j}-\Phi_{j} E_{m+k}\right)$.

Now, $X_{i}\left(E_{m+k} \Phi_{j}-\Phi_{j} E_{m+k}\right)=\left(t_{i m+k}\right) \tau_{j}-t_{i m+k}-b_{i j} D_{m+k}=b_{i j}\left(\tau_{j}^{-1} D_{m+k} \tau_{j}\right)-$ $b_{m+k j} D_{i}-b_{i j} D_{m+k}$ by (4) and $X_{i} \Phi_{j} I_{b_{m+k j}}=\left(X_{i}+b_{i j}\right) b_{m+k j}-b_{m+k j}\left(X_{i}+b_{i j}\right)=$ $b_{i j} b_{m+k j}-b_{m+k j} b_{i j}-b_{m+k j} D_{i}=\left(b_{i j} \tau_{j}^{-1}\right) \tau_{j} I_{b_{m+k j}}-b_{m+k j} D_{i}=\left(b_{i j} \tau_{j}^{-1}\right)\left(D_{m+k} \tau_{j}-\right.$ $\left.\tau_{j} D_{m+k}\right)-b_{m+k j} D_{i}=b_{i j}\left(\tau_{j}^{-1} D_{m+k} \tau_{j}\right)-b_{i j} D_{m+k}-b_{m+k j} D_{i}$ by (1). Hence we have $X_{i}\left(E_{m+k} \Phi_{j}-\Phi_{j} E_{m+k}\right)=\left(X_{i} \Phi_{j}\right) I_{b_{m+k j}}$.

Let us assume that $Y\left(E_{m+k} \Phi_{j}-\Phi_{j} E_{m+k}\right)=\left(Y \Phi_{j}\right) I_{b_{m+k j}}$ for each $Y=X_{m+k}^{\nu_{n+1}+k-1}$ $\cdots X_{1}^{\nu_{1}} t_{\nu_{m+k-1} \cdots \nu_{1}} \in T\left[\mathfrak{X}_{m+k-1} ; \mathfrak{D}_{m+k-1}\right]$ such that deg $Y=q$. Then, for each $Y=$ $X_{m+k-1}^{\nu_{m+k} \cdots X_{i}^{\prime}} \cdots X_{i}^{\nu_{i}+i} \cdots X_{1}^{\nu} t_{\nu_{n+k=1} \cdots \nu_{1}}, Y\left(E_{m+k} \Phi_{j}-\Phi_{j} E_{m+k}\right)=Y_{1}\left(E_{m+k} \Phi_{j}-\Phi_{j} E_{m+k}\right)\left(Y_{2} \Phi_{j}\right)$ $+\left(Y_{1} \Phi_{j}\right)\left(Y_{2}\left(E_{m+k} \Phi_{j}-\Phi_{j} E_{m+k}\right)\right)=\left(Y_{1} \Phi_{j} I_{b_{m+k j}}\right)\left(Y_{2} \Phi_{j}\right)+\left(Y_{1} \Phi_{j}\right)\left(Y_{2} \Phi_{j} I_{b_{m+k j}}\right)=$

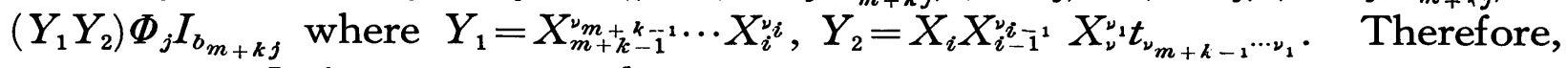
we can see $\Phi_{j}$ is an automorphism.

$X_{i} \Phi_{j} \Phi_{k}=X_{i}+b_{i k}+b_{i j} \tau_{k}, \quad X_{i} \Phi_{k} \Phi_{j}=X_{i}+b_{i j}+b_{i k} \tau_{j}$ shows that $\Phi_{j} \Phi_{k}=\Phi_{k} \Phi_{. j}$ if and only if there holds (5). 
$X_{i} \Phi_{j}^{\# \tau_{j}}=X_{i}+T_{\# \tau_{j}}\left(b_{i j} ; \tau_{j}\right)$ shows that $(2)$ is equivalent with $\# \Phi_{j}=p^{f_{j}+j}$ if $j \leqq m$ and $\# \Phi_{j}=p$ if $j>m$. Now $\left(X_{i}^{\mathfrak{p}}-t_{i}\right) \Phi_{j}=X_{i}^{\mathfrak{p}}+\left(\Delta_{p}^{(i)}\left(b_{i j}\right)-b_{i j}\right)-t_{i} \tau_{j}$ shows that (3) is equivalent with that $X_{i}^{p}-t_{i}$ is left invariant under $\Phi_{j}$. Thus each $\Phi_{j}$ induces an automorphism $\sigma_{j}^{*}$ of order $p^{f_{j}+1}$ if $j \leqq m$, of order $p$ if $j>m$ in $R^{*}=T\left[y_{1}, y_{2}, \cdots, y_{m+k}\right] \cong T\left[\mathfrak{X}_{m+k} ; \mathfrak{D}_{m+k}\right] / M$ where $R^{*}$ is a $p^{f+k+m}$-dimensional simple ring extension over $S$.

Let $\sigma \in\left(\sigma_{j}^{*}\right)_{\cap}\left(\sigma_{1}^{*}, \sigma_{2}^{*}, \cdots, \sigma_{j-1}^{*}\right)$. If $\sigma=\sigma_{j}^{* \alpha_{j}}=\sigma_{1}^{* \alpha_{1}} \sigma_{2}^{* \alpha_{2}} \cdots \sigma_{j-1}^{* \alpha_{j-1}}$, we may assume that each $\alpha_{i}$ is a multiple of each \# $\tau_{i}$ since $1=\sigma \mid T=\tau_{j}^{\alpha_{j}}=\tau_{1}^{\alpha_{1}} \tau_{2}^{\alpha_{2}} \cdots \tau_{j}^{\alpha_{j}} \overline{1}^{1}$, Then $y_{j} \sigma=y_{j}+T_{\alpha_{j}}\left(b_{j j} ; \tau_{j}\right)=y_{j}+\alpha_{j} / \# \tau_{j}=y_{j} \sigma_{1}^{* \alpha_{1}} \sigma_{2}^{* \alpha_{2}} \cdots \sigma_{j-1}^{\alpha_{j}{ }^{1}}=y_{j}$ yields at once $\sigma=1$. Hence $\mathbb{S B}^{*}$, the group generated by $\sigma_{1}^{*}, \sigma_{2}^{*}, \cdots, \sigma_{m+k}^{*}$, is coincides with the direct product of them. Finally, $S \subseteq J\left(\mathbb{S}^{*}, R^{*}\right)=J\left(\mathbb{S}^{*} \mid T, T\right)=S$. Thus the rest, we shall show that $\mathbb{S B}^{*}$ is an $F$-group.

Firstly, we note that $C^{*}[Z]$ is a commutative semi-simple artinian ring as same reason as that of Theorem 3.1. Let $\left\{\widetilde{\tau}_{1}, \cdots, \widetilde{v}_{m}\right\}=\mathbb{S}^{*}{ }_{n} \widetilde{V}^{*}$. Then $v_{i} \sigma_{j}^{*}=c_{i j} v_{i}$ for some $c_{i j} \in C^{*}$ such that $N_{\sharp \sigma_{j}^{*}}\left(c_{i j} ; \sigma_{j}^{*}\right)=1$ and $v_{i} v_{j}=v_{j} v_{i}$. Thus, we can see that $C^{*}\left[v_{1}, v_{2}, \cdots, v_{m}\right] \equiv C^{*}[Z]$. If $v=\sum c_{\alpha} w_{\alpha}, c_{\alpha} \in C^{*}$ and $w_{\alpha}=$ $v_{m}^{\beta_{m}} v_{m-1}^{\beta_{m}} \cdots v_{1}^{\beta_{1}}$, is an arbitrary element of $C^{*}\left[v_{1}, v_{2}, \cdots, v_{m}\right]$, then $v^{p^{n}} \in C^{*}$ for some positive integer $a$. Thus $C^{*}\left[v_{1}, v_{2}, \cdots, v_{m}\right]$ is a field.

Conversely, let $T / S$ can be regularly embedded in a $p^{f+k+m}$-dimensional abelian extension $R / S$ with respect to $\mathbb{S}=\left(\sigma_{1}\right) \times\left(\sigma_{2}\right) \times \cdots \times\left(\sigma_{m}\right) \times\left(\sigma_{m+1}\right) \times \cdots \times$ $\left(\sigma_{m+k}\right)$ if $k \geqq 0$ and $\mathbb{S}=\left(\sigma_{1}\right) \times \cdots \times\left(\sigma_{m+k}\right) \times\left(\tau_{m+k+1}\right) \times \cdots \times\left(\tau_{m}\right)$ if $-m<k<0$ such that $\# \sigma_{j}=\left(\# \tau_{j}\right) p, \sigma_{j} \mid T=\tau_{j}$ if $j \leqq m$ and $\# \sigma_{j}=p, \sigma_{j} \mid T=1$ if $j>m$. Since $V$ is a field and $[R: T]=p^{m+k}, R / T$ is a fundamental abelian extension with respect to $\mathbb{S S}(T)=\left(\sigma_{1}^{p^{f}}\right) \times \cdots \times\left(\sigma_{m}^{p^{f} m}\right) \times\left(\sigma_{m+1}\right) \times \cdots \times\left(\sigma_{m+k}\right)$. Then $R=\underset{0 \leq \nu_{j}<p}{\bigoplus} x_{e}^{\nu} x_{e-1}^{\nu} e_{-1}^{1} \cdots x_{1}^{\nu_{1}} T$, $x_{i} \sigma_{j}^{p_{j} j}-x_{i}=\delta_{i j}$ if $j \leqq m$ and $x_{i} \sigma_{j}-x_{i}=\delta_{i j}$ if $j>m$. Evidently $b_{i j}=x_{i} \sigma_{j}-x_{i}$ is contained in $T$ and $T_{\# r_{j}}\left(b_{i j} ; \tau_{j}\right)=\sum_{k=\sigma_{j}-1}^{\#_{k}}\left(x_{i} \sigma_{j}-x_{i}\right) \sigma_{j}^{k}=\delta_{i j}$, further, $b_{i j}\left(\tau_{k}-1\right)$ $=\left(x_{i} \sigma_{j}-x_{i}\right)\left(\sigma_{k}-1\right)=\left(x_{i} \sigma_{j}-x_{i}\right) \sigma_{k}-\left(x_{i} \sigma_{j}-x_{i}\right)=\left(x_{i} \sigma_{k}-x_{i}\right) \sigma_{j}-\left(x_{i} \sigma_{k}-x_{i}\right)=$ $b_{i k}\left(\tau_{j}-1\right)$.

Now, we set $D_{i}=I_{x_{i}}, t_{i}=x_{i}^{p}-x_{i}$ and $t_{i j}=x_{i} x_{j}-x_{j} x_{i}$. Then one will easily check that $\left\{D_{1}, D_{2}, \cdots, D_{m+k},\left(t_{i}\right),\left(t_{i j}\right)\right\}$ satisfies the conditions (1)-(5) of Theorem 3.1 in $T$.

$$
\begin{aligned}
& \quad t\left(D_{i} \tau_{j}-\tau_{j} D_{i}\right)=\left(t x_{i}-x_{i} t\right) \tau_{j}-\left(t \tau_{j} x_{i}-x_{i} t \tau_{j}\right)=t \tau_{j}\left(x_{i} \sigma_{j}-x_{i}\right)-\left(x_{i} \sigma_{j}-x_{i}\right) t \tau_{j} \\
& =t \tau_{j} I_{b_{i j}} \quad(t \in T) . \\
& \quad t_{i j}\left(\tau_{k}-1\right)=\left(x_{i} x_{j}-x_{j} x_{i}\right) \sigma_{k}-\left(x_{i} x_{j}-x_{j} x_{i}\right)=\left(x_{i} x_{j}\right) \sigma_{k}-x_{i}\left(x_{j} \sigma_{k}\right)-\left(x_{j} x_{i}\right) \sigma_{k}+ \\
& x_{j} \sigma_{k} x_{i}-\left(x_{j} \sigma_{k}-x_{j}\right) x_{i}+x_{i}\left(x_{j} \sigma_{k}-x_{j}\right)=\left(x_{i} \sigma_{k}-x_{i}\right) x_{j} \sigma_{k}-x_{j} \sigma_{k}\left(x_{i} \sigma_{k}-x_{i}\right)-b_{j k} D_{i} \\
& =b_{i k} \tau_{k}^{-1} D_{j} \tau_{k}-b_{j k} D_{i} .
\end{aligned}
$$

Finally, $\left(x_{i}^{p}-x_{i}\right) \sigma_{j}=x_{i}^{p}+\Delta_{p}^{(i)}\left(b_{i j}\right)-x_{i}-b_{i j}$ shows that $\Delta_{p}^{(i)}\left(b_{i j}\right)-b_{i j}=\left(x_{i}^{p}-\right.$ $\left.x_{i}\right)\left(\sigma_{j}-1\right)=t_{i}\left(\tau_{j}-1\right)$. 
The map $\varphi^{*}: \sum X_{e}^{\nu_{e}} \cdots X_{1}^{\nu_{1}} t_{\nu_{e} \cdots \nu_{1}} \rightarrow \sum y_{e}^{\nu} \cdots y_{1}^{\nu} t_{v_{e} \cdots \nu_{1}}\left(t_{\nu_{e} \cdots \nu_{1}} \in T\right)$ is a $T$-isomorphism of $R^{*}$ onto $R$ satisfying $\varphi^{*} \sigma_{i}=\sigma_{i}^{*} \varphi^{*}$.

Combining Corollary 3.3 with Theorem 3.2, we can easily obtain

Corollary 3.5. Let $T / S$ be a p-dimensional abelian division ring extension of $\chi(S)=p$ with respect to $\mathfrak{Y}=\left(\tau_{1}\right) \times\left(\tau_{2}\right) \times \cdots \times\left(\tau_{m}\right)$ such that $\# \tau_{i}=p^{f_{i}}$. In order that $T / S$ can be regularly embedded in a $p^{f+k+m}$-dimensional abelian division ring extension such that $k \geqq-m$, it is necessary and sufficient that there exist derivations $D_{1}, D_{2}, \cdots, D_{m+k}$ in $T,\left(t_{i j}\right)$ an $(m+k) \times(m+k)$ matrix with entries in $T,\left(t_{i}\right)$ a $1 \times(m+k)$ matrix with entries in $T$ satisfying the conditions (1)-( $\left.5^{\prime}\right)$ of Corollary 3.3 (in $\left.T\right)$ and $\left(b_{i j}\right)$ an $(m+k) \times(m+k)$ matrix with entries in $T$ satisfing the conditions (1)-(5) of Theorem 3.2.

\section{§4. Kummer extensions.}

Throughout the present section, we assume that $Z$ contains $\zeta$, a primitive $n$-th root of 1 . An $n$-dimensional abelian extension $R$ over $S$ is called an $n$-dimensional Kummer extension if $C \ni \zeta$ and there exists a Galois group (S) $=\left(\sigma_{1}\right) \times\left(\sigma_{2}\right) \times \cdots \times\left(\sigma_{e}\right)$, a direct product of $\sigma_{i}$ with $\# \sigma_{i}=n_{i}, \prod_{i=1}^{e} n_{i}=n$ and \&S is a $D F$-group.

One of principal theorem of this section is the following

Theorem 4.1. In order that $S$ have an n-dimensional Kummer extension, it is necessary and sufficient that there exist automorphisms $\rho_{1}, \rho_{2}$, $\cdots, \rho_{e}$ in $S,\left(s_{i j}\right)$ an $e \times e$ matrix with entries in $S^{*}$ such that $s_{i j}=s_{j i}^{-1}, s_{i i}=1$ and $\left(s_{i}\right)$ a $1 \times e$ matrix with entries in $S^{\bullet}$ satisfying

(1) $\boldsymbol{\rho}_{j}^{-1} \boldsymbol{\rho}_{i}^{-1} \boldsymbol{\rho}_{j} \boldsymbol{\rho}_{i}=\widetilde{s}_{i j}$,

(2) $s_{i j}\left(s_{i k} \rho_{j}\right) s_{j k}=\left(s_{j k} \rho_{i}\right) s_{i k}\left(s_{i j} \rho_{k}\right)$,

(3) $\boldsymbol{\rho}_{i}^{n_{i}}=\widetilde{s}_{i}^{-1}$ such that $\prod_{i=1}^{e} n_{i}=n, s_{i} \rho_{i}=s_{i}$ and $\zeta \rho_{i}=\zeta$,

(4) $R N_{n_{i}}\left(s_{j i} ; \boldsymbol{\rho}_{i}\right)=\left(s_{i}^{-1} \rho_{j}\right) s_{i}$,

(5) $f\left(X_{\pi(i)}\right)=X_{\pi(i)}^{n_{\pi(i)}}-s_{\pi(i)}$ is s.w-irreducible in $S\left[\mathfrak{X}_{\pi(e)} ; \mathfrak{P}_{\pi(e)}\right]$,

(6) If $g(X)$ is a polynomial of $S\left[\mathfrak{X}_{\pi(e)} ; \mathfrak{F}_{\pi(e)}\right] \backslash M, M=\left(f\left(X_{1}\right)\right.$, $\left.f\left(X_{2}\right), \cdots, f\left(X_{e}\right)\right)$, then $\sum_{i} f_{i}(X) g(X) u_{i}=1$ modulo $M$ for some $f_{i}(X)$ $\in S\left[\mathfrak{X}_{\pi(e)} ; \mathfrak{P}_{\pi(e)}\right], u_{i} \in S$.

More precisely, if there exists $\left\{\rho_{1}, \rho_{2}, \cdots, \rho_{e},\left(s_{i j}\right),\left(s_{i}\right)\right\}$ satisfying (1)-(6), then $M=\left(f\left(X_{1}\right), f\left(X_{2}\right), \cdots, f\left(X_{e}\right)\right) S\left[\mathfrak{X}_{e} ; \mathfrak{B}_{e}\right]$ is maximal and $R^{*}=S\left[y_{1}, y_{2}, \cdots, y_{e}\right]$ $\cong S\left[\mathfrak{X}_{e} ; \mathfrak{P}_{e}\right] / M, y_{i}$ is the residue class of $X_{i}$ modulo $\mathrm{M}$, is an $n$-dimensional Kummer extension with generating automorphisms $\sigma_{1}^{*}, \sigma_{2}^{*}, \cdots, \sigma_{e}^{*}$ such that $x_{i} \sigma_{j}^{*} \begin{cases}=x_{i} \zeta^{n / n_{i}} & \text { if } i=j \\ =x_{i} & \text { if } i \neq j .\end{cases}$ 
Conversely, if $R$ is an n-dimensional Kummer extension over $S$ with respect to $\mathbb{S}=\left(\sigma_{1}\right) \times\left(\sigma_{2}\right) \times \cdots \times\left(\sigma_{e}\right)$, \# $\sigma_{i}=n_{i}$ and $\prod_{i=1}^{e} n_{e}=n$, then we can find such $\left\{\rho_{1}, \rho_{2}, \cdots, \rho_{e},\left(s_{i j}\right),\left(s_{i}\right)\right\}$ satisfying $(1)-(5)$ and $S\left[\mathfrak{X}_{e} ; \mathfrak{P}_{e}\right]$ satisfying $(6)$ that there holds an $S$-isomorphism $\varphi^{*}: R^{*} \cong R$ with $\varphi^{*} \sigma_{i}=\sigma_{i}^{*} \varphi^{*}$ for each $i=1,2, \cdots, e$.

Proof. By Proposition 2.2, there exists a polynomial ring $S\left[\mathfrak{X}_{e} ; \mathfrak{B}_{e}\right]=$ $\left\{\sum X_{e}^{\nu} X_{e-1}^{\nu} \cdots X_{1}^{\nu_{1}} s_{\nu_{e} \cdots \nu_{1}} ; s_{\nu_{e} \cdots \nu^{1}} \in S\right\}$ such that $s X_{i}=X_{i}\left(s \rho_{i}\right), X_{i} X_{j}=X_{j} X_{i} s_{i j}((1)$ and (2)).

Since $X_{j}\left(X_{i}^{n_{i}} s_{i}^{-1}-1\right)=X_{i}^{n_{i}} X_{j} R N_{n_{i}}\left(s_{j i} ; \rho_{i}\right) s_{i}^{-1}-X_{j}$, (4) means that $X_{j}\left(X_{i}^{n_{i}} s_{i}^{-1}\right.$ $-1)=\left(X_{i}^{n_{i}} s_{i}^{-1}-1\right) X_{j}$. Thus, with the first two conditions of (3), we can see that $M=\left(f\left(X_{1}\right), f\left(X_{2}\right), \cdots, f\left(X_{e}\right)\right) S\left[\mathfrak{X}_{e} ; \mathfrak{P}_{e}\right]$ is a two-sided ideal, and by (5), it is maximal.

Now, let $\Psi_{i}(i=1,2, \cdots, e)$ be the map of $S\left[\mathfrak{X}_{e} ; \mathfrak{F}_{e}\right]$ defined by $\sum X_{e}^{\nu} X_{e-1}^{\nu}$ $\cdots X_{i}^{\nu} \cdots X_{1}^{\nu_{1}} S_{\nu_{e} \cdots \nu_{1}} \rightarrow \sum X_{e}^{\nu} X_{e-1}^{\nu_{e}{ }^{1}} \cdots\left(X_{i} \zeta^{n / n}\right)^{\nu_{i}} \cdots X_{1}^{\nu_{1}} s_{\nu_{e} \cdots \nu_{1}}$. Then $\Psi_{i}$ is an automorphism. For, $f \Psi_{i} X_{i} \Psi_{i}=f \cdot X_{i} \zeta^{n / n_{i}}=X_{i}\left(f P_{i}\right) \zeta^{n / n_{i}}$ and $\left[X_{i}\left(f P_{i}\right)\right] \Psi_{j}=X_{i} \zeta^{n / n_{i}} f P_{i}=$ $X_{i}\left(f P_{i}\right) \zeta^{n / n_{i}}$ (by the last condition of $\left.(3)\right)$ for each $f \in S\left[X_{1}, \cdots, \check{X}_{i}, \cdots, X_{e}\right.$; $\left.P_{1}, \cdots, \breve{P}_{i}, \cdots, P_{e}\right]$. Hence $\Psi_{i}$ is a ring epimorphism, and $\# \Psi_{i}=n_{i}$ shows that $\Psi_{i}$ is an automorphism.

$\left(X_{i}^{n_{i}}-s_{i}\right) \Psi_{i}=\left(X_{i} \zeta^{n / n_{i}}\right)^{n_{i}}-s_{i}=X_{i}^{n_{i}}-s_{i}$ implies that $M \Psi_{i}=M$. Thus each $\Psi_{i}$ induces an automorphism $\sigma_{i}^{*}$ of order $n_{i}$ in $R^{*}=S\left[y_{1}, y_{2}, \cdots, y_{e}\right]$ where $R^{*}$ is an $n$-dimensional simple ring over $S$ which is isomorphic to $S\left[\mathfrak{X}_{e} ; \mathfrak{P}_{e}\right] / M$ and $y_{i}$ is the residue class of $X_{i}$ modulo $M$.

Let $\sigma \in\left(\sigma_{i}^{*}\right)_{\bigcap}\left(\sigma_{1}^{*}, \sigma_{2}^{*}, \cdots, \sigma_{i-1}^{*}\right)$. If $\sigma=\sigma_{i}^{* \alpha_{i}}=\sigma_{1}^{* \alpha_{1}} \sigma_{2}^{* \alpha_{2}} \cdots \sigma_{i-1}^{* \alpha_{i-1}}, x_{i} \sigma_{i}^{*}=x_{i} \zeta^{k n / n_{i}}=$ $x_{i} \sigma_{1}^{* \alpha_{1}} \sigma_{2}^{* \alpha_{2}} \cdots \sigma_{i-1}^{* \alpha_{i-1}}=x_{i}$ shows that $\sigma=1$. Hence (S), the group generated by $\sigma_{1}^{*}, \sigma_{2}^{*}, \cdots, \sigma_{e}^{*}$, coincides with a direct product of them since $\sigma_{i}^{*} \sigma_{j}^{*}=\sigma_{j}^{*} \sigma_{i}^{*}$ is clear. Thus we have $J\left(S^{*}, R^{*}\right)=\bigcap_{i=1}^{e} J\left(\sigma_{i}^{*}, R^{*}\right)=S$.

The condition (6) is equivalennt with that $R^{*}$ is $R_{l}^{*} S_{r}$-irreducible. Hence, by Proposition $1.2, V^{*}$ is a division ring.

Conversely, if $R$ is an $n$-dimensional Kummer extension over $S$ with

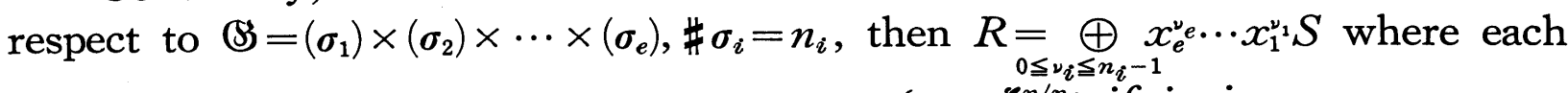
$x_{i}$ is an element contained in $R^{\bullet}$ and $x_{i} \sigma_{j}\left\{\begin{array}{ll}=x \zeta^{n / n_{i}} & \text { if } i=j \\ =x_{i} & \text { if } i \neq j\end{array}\right.$ [Theorem 4 of [8]].

We set $\rho_{i}=\widetilde{x}_{i}^{-1} \mid S, s_{i}=x_{i}^{n_{i}}, s_{i j}=x_{i}^{-1} x_{j}^{-1} x_{i} x_{j} . \quad$ Then $\left(x_{i}^{-1} s \cdot x_{i}\right) \sigma_{j}=x_{i}^{-1} s x_{i}$, $x_{i}^{n_{i}} \sigma_{j}=x_{i}^{n_{i}}$ and $s_{i j} \sigma_{k}=s_{i j}$ show that $\rho_{i}$ is an automorphism of $S, s_{i}, s_{j i} \in S$ respectively, and $\rho_{i}$ satisfies (3).

Now $s \boldsymbol{\rho}_{j}^{-1} \boldsymbol{\rho}_{i}^{-1} \boldsymbol{\rho}_{j} \boldsymbol{\rho}_{i}=\left(x_{j} s x_{j}^{-1}\right) \boldsymbol{\rho}_{i}^{-1} \boldsymbol{\rho}_{j} \boldsymbol{\rho}_{i}=\left(x_{i} x_{j} s x_{j}^{-1} x_{i}^{-1}\right) \boldsymbol{\rho}_{j} \boldsymbol{\rho}_{i}=x_{i}^{-1} x_{j}^{-1} x_{i} x_{j} s x_{j}^{-1} x_{i}^{-1} x_{j} x_{i}$ $=s \tilde{s}_{i j}$ for each $s \in S$. 
$\left(s_{i j}\right)\left(s_{i k} \rho_{j}\right) s_{j k}=\left(x_{i}^{-1} x_{j}^{-1} x_{i} x_{j}\right)\left(x_{j}^{-1} x_{i}^{-1} x_{k}^{-1} x_{i} x_{k} x_{j}\right)\left(x_{j}^{-1} x_{k}^{-1} x_{j} x_{k}\right)=x_{i}^{-1} x_{j}^{-1} x_{k}^{-1} x_{i} x_{j} x_{k}$. On ther other hand, $\left(s_{j k} \rho_{i}\right) s_{i k}\left(s_{i j} \rho_{k}\right)=\left(x_{i}^{-1} x_{j}^{-1} x_{k}^{-1} x_{j} x_{k} x_{i}\right)\left(x_{i}^{-1} x_{k}^{-1} x_{i} x_{k}\right)$ $\left(x_{k}^{-1} x_{i}^{-1} x_{j}^{-1} x_{i} x_{j} x_{k}\right)=\left(x_{i}^{-1} x_{j}^{-1} x_{k}^{-1} x_{j} x_{i} x_{k}\right)\left(x_{k}^{-1} x_{i}^{-1} x_{j}^{-1} x_{i} x_{j} x_{k}\right)=x_{i}^{-1} x_{j}^{-1} x_{k}^{-1} x_{i} x_{j} x_{k}$. Thus there holds (2).

Next, $R N_{n_{i}}\left(s_{j i} ; \boldsymbol{\rho}_{i}\right)=\left(s_{j i}\right)\left(s_{j i} \boldsymbol{\rho}_{i}\right) \cdots\left(s_{j i} \boldsymbol{\rho}_{i}^{n_{i-1}}\right)$ $=\left(s_{j i}\right)\left(x_{i}^{-1} s_{j i} x_{i}\right)\left(x_{i}^{-2} s_{j i} x_{i}^{2}\right) \cdots\left(x_{i}^{1-n_{i}} s_{j i} x_{i}^{n_{i-1}}\right)=\left(s_{j i} x_{i}^{-1}\right)^{n_{i-1}}\left(s_{j i} x_{i}^{n_{i-1}}\right)$ $=\left(x_{j}^{-1} x_{i}^{-1} x_{j}\right)^{n_{i-1}}\left(x_{j}^{-1} x_{i}^{-1} x_{j} x_{i}^{n}\right)=\left(x_{j}^{-1} x_{i}^{1-n_{i}} x_{j}\right)\left(x_{j}^{-1} x_{i}^{-1} x_{j} x_{i}^{n_{i}}\right)=x_{j}^{-1} x_{i}^{-n_{i}} x_{j} x_{i}^{n_{i}}=\left(s_{i}^{-1} \rho_{j}\right) s_{i}$.

Since $R=S\left[x_{1}, x_{2}, \cdots, x_{e}\right] \cong S\left[\mathfrak{X}_{e} ; \mathfrak{P}_{e}\right] / M, M=\left(X_{1}^{n_{1}}-x_{1}^{n_{1}}, X_{2}^{n_{2}}-x_{2}^{n_{2}}, \cdots, X_{e}^{n_{e}}\right.$ $\left.-x_{e}^{n_{e}}\right) S\left[\mathfrak{X}_{e} ; \mathfrak{B}_{e}\right], X_{i} P_{j}=X_{i} s_{i j}, S \mid P_{j}=\rho_{j}$, and $V$ is a division ring, each $X_{i}^{n_{i}}-x_{i}^{n_{i}}$ is $s . w$-irreducible in $S\left[\mathfrak{X}_{e} ; \mathfrak{P}_{e}\right]$ and $S\left[\mathfrak{X}_{e} ; \mathfrak{P}_{e}\right]$ satisfies (6).

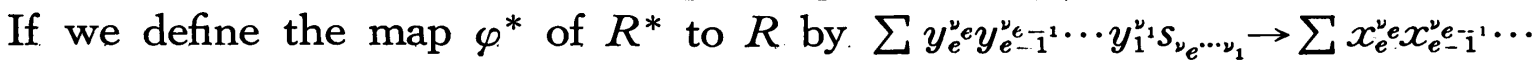
$x_{1}^{\nu_{1}} s_{\nu_{e} \cdots \nu_{1}}, \varphi^{*}$ is an $S$-isomorphism satisfying $\sigma_{i}^{*} \varphi^{*}=\varphi^{*} \sigma_{i}$ for each $i=1,2, \cdots, e$.

Corollary 4. 1. (a) In order that $S$ have an n-dimensional outer Kummer extension, it is necessary and sufficient that there exist $\left\{\rho_{1}, \rho_{2}, \cdots, \rho_{e},\left(s_{i j}\right),\left(s_{i}\right)\right\}$ satisfying the conditions (1)-(5) of Theorem 4.1 and if $\rho_{e}^{\nu} \rho_{e-1}^{\nu} e_{-1}^{1} \ldots \rho_{1}^{\nu}=\widetilde{s}$ for some $s \in S$, then,

(7) $s \boldsymbol{\rho}_{i}=R N_{\nu_{1}}\left(s_{i 1} ; \rho_{1}\right)\left\{R N_{\nu_{2}}\left(s_{i_{2}} ; \boldsymbol{\rho}_{2}\right)\right\} \boldsymbol{\rho}_{1}^{\nu_{1}}\left\{R N_{\nu_{3}}\left(s_{i 3} ; \rho_{3}\right)\right\} \rho_{2}^{\nu_{2}} \rho_{1}^{\nu_{1}} \ldots$ $R N_{v_{e}}\left(s_{i e} ; \rho_{e}\right) \rho_{e-1}^{\nu} e_{-1}^{1} \ldots \rho_{1}^{\nu} s^{\nu}$.

(b) In order that $S$ have an n-dimensional inner Kummer extension it is necessary and sufficient that there exist $\left\{\rho_{1}, \rho_{2}, \cdots, \rho_{e},\left(s_{i j}\right),\left(s_{i}\right)\right\}$ satisfying (1)-(6) of Theorem 4.1 and there exist a $1 \times e$ matrix $\left(t_{i}\right)$ with entries in $T$ satisfying

(7') $\rho_{e}^{\nu} e i \ldots \rho_{1}^{\nu_{1} i}=\tilde{\boldsymbol{t}}_{i}$ $t_{i} \rho_{i}=R N_{\nu_{1 i}}\left(s_{i 1} ; \rho_{1}\right)\left\{R N_{\nu 2_{i}}\left(s_{i 2} ; \rho_{2}\right)\right\} \rho_{1}^{\nu_{1 i}}\left\{R N^{\nu_{3 i}}\left(s_{i 3} ; \rho_{3}\right)\right\} \rho_{2}^{\nu_{2 i}} \rho_{1}^{\nu_{1} i z} \ldots$ $\left.\left\{R N_{v_{e j}}\left(s_{i e} ; \rho_{e}\right)\right\} \rho_{e-1}^{v_{e}} \ldots \rho_{1}^{\nu_{1}} t_{i}\right\}^{n / n_{i}}$

$t_{i} \rho_{j}=R N_{\nu_{1 i}}\left(s_{i 1} ; \rho_{1}\right)\left\{R N_{\nu_{2 i}}\left(s_{i 2} ; \rho_{2}\right)\right\} \rho_{1_{1}^{\nu_{1} i z}}^{\nu_{12}}\left\{R N_{\nu_{3 i}}\left(s_{i 3} ; \rho_{3}\right)\right\} \rho_{2}^{\nu_{2 i} i} \rho_{1}^{\nu_{1 i}} \ldots$ $\left\{R N_{\nu_{e i}}\left(s_{i e} ; \rho_{e}\right)\right\} \rho_{e-1}^{\nu_{e}-1 i} \ldots \rho_{1}^{\nu_{1} i}(i \neq j)$.

In particular.

(c) In order that $S$ have an n-dimensional inner Kummer extension such that $V=Z$, it is necessary and sufficient that there exist $\left\{\rho_{1}, \rho_{2}, \cdots, \rho_{e}\right.$, $\left.\left(s_{i j}\right),\left(s_{i}\right)\right\}$ satisfying (1)-(4) of Theoem 4.1 and there exist $a 1 \times n$ matrix $\left(z_{i}\right)$ with entries in $Z^{*}$ satisfying

(7') $z_{i} \rho_{j} \begin{cases}=z_{i} \zeta^{n / n_{i}} & \text { if } i=j \\ =z_{i} & \text { if } i \neq j .\end{cases}$

Proof. If there exists $\left\{\boldsymbol{\rho}_{1}, \boldsymbol{\rho}_{2}, \cdots, \boldsymbol{\rho}_{e},\left(s_{i j}\right),\left(s_{i}\right)\right\}$ satisfying the conditions (1)(5) of Theorem 4.1 , then there exists an $n$-dimensional simple ring extension $R^{*}=S\left[y_{1}, y_{2}, \cdots, y_{e}\right]$ over $S$ such that $J\left(\mathbb{S}^{*}, R^{*}\right)=S$.

(a) Let $v=\sum y_{e}^{\nu} y_{e-1}^{\nu} e_{-1}^{1} \cdots y_{1}^{\nu_{1}} s_{v_{e} \cdots \nu_{1}}$ be an element of $V^{*}$. Then $s v=v s(s \in S$ 


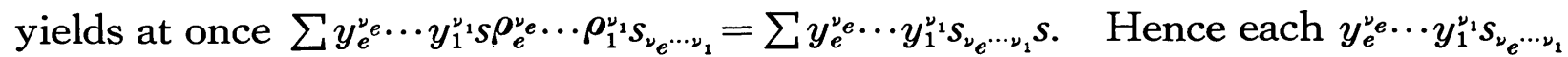
is contained in $V^{*}$ and $\rho_{e}^{\nu} e_{1}^{\nu} \rho_{1}^{\nu}=\widetilde{s}_{\nu_{e} \cdots \nu_{1}}$ since $S=S s_{\nu_{e} \cdots \nu_{1}} S=S \rho_{e}^{\nu} e_{e} \ldots \rho_{1}^{\nu} s_{\nu_{e} \cdots \nu_{1}} S=s_{\nu_{e} \cdots \nu_{1}} S$. Thus $\rho_{e}^{y_{e}} \ldots \rho_{1}^{\nu_{1}}=\widetilde{s}$ if and only if $y_{e}^{\nu_{e}} \ldots y_{1}^{\nu_{1}} s$ is contained in $V^{*}$. Now, let $v=$ $y_{e}^{\nu} \omega_{1} y_{1}^{\nu_{1}} s$ be in $V^{*}$. Then $v \in C^{*}$ if and only if $y_{i} v=v y_{i}$ for each $y_{i}$, and, that is $R N_{\nu_{1}}\left(s_{i 1} ; \rho_{1}\right)\left\{R N_{\nu_{2}}\left(s_{i 2} ; \rho_{2}\right)\right\} \rho_{1}^{\nu_{1}}\left\{R N_{\nu_{3}}\left(s_{i 3} ; \rho_{3}\right)\right\} \rho_{2}^{\nu_{2}} \rho_{1}^{\nu_{1}} \ldots\left\{R N_{\nu_{e}}\left(s_{i e} ; \rho_{e}\right)\right\} \rho_{e-1}^{\nu_{e}} e_{1}^{1} \ldots$ $\rho_{1}^{\nu} s=s \rho_{i}$.

(b) As was shown in the proof of (a), $\rho_{e}^{\nu} e \ldots \rho_{1}^{\nu}=\tilde{t}_{i}$ is equivalent with $v_{i}=y_{e}^{v^{e} i \cdots y_{1}^{\nu_{1} i} t_{i}}$ is contained in $V^{*}$. Hence if $t_{i}$ satisfies the two conditions of $\left(7^{\prime}\right)$, then $v_{i} y_{i}=y_{e}^{\nu} e \cdot \cdots y_{1}^{\nu_{1} i} y_{i} t_{i} \rho_{i}=y_{e}^{\nu} e_{i} \cdots y_{1}^{\nu_{1} i} y_{i} . R N_{\nu_{1 i}}\left(s_{j 1} ; \rho_{1}\right)\left\{R N_{\nu_{2 i}}\left(s_{i 1} ; \rho_{2}\right)\right\} \rho_{1}^{\nu_{1} i}$

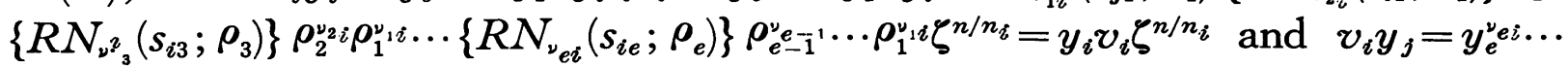

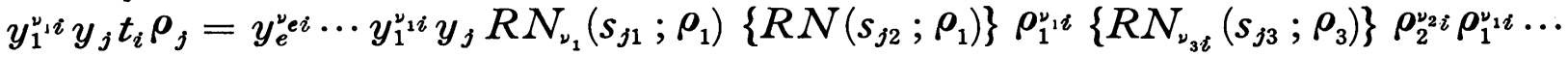
$\left\{R N_{v_{e i}}\left(s_{j e} ; \rho_{e}\right)\right\} \rho_{e-1}^{v_{e}-1} \ldots \rho_{1}^{\nu_{1} i t}=y_{j} v_{i}$ for each $j \neq i$. Consequently, we have $v_{i} y_{j} v_{i}^{-1}\left\{\begin{array}{ll}=y_{j} \zeta^{n / n_{i}} & \text { if } i=j \\ =y_{j} & \text { if } i \neq j .\end{array}\right.$ Thus we may adapt $\widetilde{v}_{i}$ as $\sigma_{i}^{*}$.

Conversely, if $R=S\left[x_{1}, x_{2}, \cdots, x_{e}\right]$ is an $n$-dimensional inner Kummer extension over $S$, then $\sigma_{i}=\tilde{v}_{i}=\overline{\sum x_{e}^{v} x_{e-1}^{\nu} e_{1}^{1} \cdots x_{1}^{\nu} t_{\nu_{e} \cdots \nu_{1}}^{(i)}}$ for each $i=1,2, \cdots, e$. Hence each $t_{v_{e} \cdots \nu_{1}}^{(i)}$ satisfies the condition $\left(7^{\prime}\right)$.

(c) If there exists $\left(z_{i}\right)$ satisfying $\left(7^{\prime \prime}\right)$, then we can prove that each $f\left(X_{i}\right)$ $=X_{i}^{n_{i}}-s_{i}$ is $s . w$-irreducible in $S\left[\mathfrak{X}_{e} ; \mathfrak{F}_{e}\right]$. For let $g\left(X_{i}\right)=\sum_{v=0}^{k} X_{i}^{v} f_{v}$ be a proper left factor of $f\left(X_{i}\right)$ in $S\left[y_{1}, \cdots, y_{i-1}\right]\left[X_{i} ; P_{i}\right]$ where $f_{\nu} \in S\left[y_{1}, \cdots, y_{i-1}\right]$. Then $f_{0} \neq 0$. Since the constant term of $z_{i} g\left(X_{i}\right)-g\left(X_{i}\right) z_{i} \zeta^{k n / n_{i}}$ is $f_{0} z_{i}\left(1-\zeta^{k n / n_{i}}\right) \neq 0$ and $\operatorname{deg}\left(z_{i} g\left(X_{i}\right)-g\left(X_{i}\right) z_{i} \zeta^{k n / n_{i}}\right)<k$, an easy induction will yield at once $\left(g\left(X_{i}\right)\right)$ $=S\left[y_{1}, \cdots, y_{i-1}\right]\left[X_{i} ; P_{i}\right]$.

Next, since $z_{i} y_{j} z_{i}^{-1}=y_{j} z_{i} \rho_{i} z_{i}^{-1}\left\{\begin{array}{ll}=y_{j} \zeta^{n / n_{i}} & \text { if } i=j \\ =y_{j} & \text { if } i \neq j\end{array}\right.$, we may regard $\left(\tilde{z}_{i}\right)$ as $\left(\boldsymbol{\sigma}_{i}^{*}\right)$. Then $V^{*}=J\left(\mathbb{S}^{*} \mid V^{*}, V^{*}\right)=Z$. The converse is almost evident.

Now as is shown in the proof of Corollary 4.1 (b), if $\rho_{i}=\widetilde{s}_{i}\left(s_{i} \in S\right)$ for each $i=1,2, \cdots, e$, then $y_{i} s_{i} \in V^{*}$ where $R^{*}=S\left[y_{1}, \cdots, y_{e}\right]$. Hence we obtain $R^{*}=S \otimes_{z} V^{*}=S\left[V^{*}\right]$. Using this fact we have

Corollary 4.2. The followings are equivalent.

(1) $S$ has an n-dimensional trivial Kummer extension.

(2) There exist elements $z_{1}, z_{2}, \cdots, z_{e}$ in $Z$ such that the associated algebraic set of the ideal $\left(X_{1}^{n_{1}}-z_{1}, X_{2}^{n_{2}}-z_{2}, \cdots, X_{e}^{n_{e}}-z_{e}\right)$ in $Z\left[X_{1}, X_{2}, \cdots, X_{e}\right]$ is an algebraic variety.

(3) There exist inner autmorphisms $\rho_{1}, \rho_{2}, \cdots, \rho_{e}$ in $S,\left(s_{i j}\right)$ an $e \times e$ matrix with entries in $S^{\bullet},\left(s_{i}\right)$ a $1 \times e$ matrix with entries in $S^{\bullet}$ satisfying (1)-(4) of Theorem 4.1 and if $\rho_{e}^{v} e \ldots \rho_{1^{\prime \prime}}=\widetilde{s}$ then $s$ satisfies $\left(7^{\prime}\right)$ of Corollary $4.1(a)$. 
Proof. The implication $(1) \rightarrow(2) \rightarrow(3)$ is easy. (3) $\rightarrow(1)$ is an immediate consequence of the remark above mentioned and Corollary 4.1 (a).

The condition (6) of Theorem 4.1 was need only to see that the centralizer $V^{*}$ is a division ring. Accordigly we have

Corollary 4.3. Let $S$ be a division ring. In order that $S$ have an n-dimensional Kummer division ring extension, it is necessary and sufficient that there exist $\left\{\rho_{1}, \rho_{2}, \cdots, \rho_{e},\left(s_{i j}\right),\left(s_{i}\right)\right\}$ satisfying (1)-(4) of Theorem 4.1 and

$\left(5^{\prime}\right) \quad f\left(X_{i}\right)=X_{i}^{n_{i}}-s_{i}$ is s-irreducible in $S\left[\mathfrak{X}_{e} ; \mathfrak{P}_{e}\right]$.

The following is corresponding to Corollary 3.4 and we can prove it as similar to that of Corollary 3.4.

Corollary 4.4. Let $S$ be a simple ring with automorphisms $\rho_{1}, \rho_{2}, \cdots, \rho_{i}$, $\left(s_{j i}\right)$ and $\left(s_{i}\right)$ satisfying the conditions (1)-(6) of Theorem 4.1.

(a) In order that $S$ have an n-dimensional parallel Kummer extension, it is necessary and sufficient that there exists a simple subring $W$ of $S$ containing $\left\{s_{j i}\right\},\left\{s_{i}\right\}$ such that $W \rho_{i} \subseteq W, V_{W}(W) \ni \zeta$ and $X_{i}^{n_{i}}-s_{i}$ is $s \cdot$.w-irreducible in $W\left[\mathfrak{X}_{e} ; \mathfrak{P}_{e}\right]$ for each $i=1,2, \cdots, e$.

(b) In order that $S$ have an n-dimensional d-parallel Kumer extension, it is necessary and sufficient that there exist a division subring $W$ of $S$ containing $\left\{s_{i j}\right\},\left\{s_{i}\right\}$ such that $W \rho_{i} \leqq W, V_{W}(W) \in \zeta$ and $X_{i}^{n_{i}}-s_{i}$ is s-irreducible in $W\left[\mathfrak{X}_{e} ; \mathfrak{P}_{e}\right]$ for each $i=1,2, \cdots, e$.

(c) In order that $S$ have an n-dimensional f-parallel Kummer extension, it is necessary and sufficient that there exist a subfield $W$ of $S$ containing $\left\{s_{i j}\right\},\left\{s_{i}\right\}$ such that $\rho_{i} \mid W=1, W \ni \zeta$ and $X_{i}^{n_{i}}-s_{i}$ is s-irreducible in $W\left[\mathfrak{X}_{e} ; \mathfrak{B}_{e}\right]$ for each $i=1,2, \cdots, e$.

Finally, we shall deal with the regular embedding problem of Kummer case.

Lemma 4.1. Let $T$ be a q-dimensional simple ring extension over $S$ such that $T$ is $T_{l} S_{r}$-irreducible and $V_{T}(T) \ni \zeta$, and let $R$ be an n-dimensional Kummer extension over $T$. Then $R$ is $R_{l} S_{r}$-irreducible if and only if $V$ is a division ring.

Proof. Let $R=T\left[x, \cdots, x_{e}\right], x_{i}^{n_{i}} \in T$, and let $V$ be a division ring. We

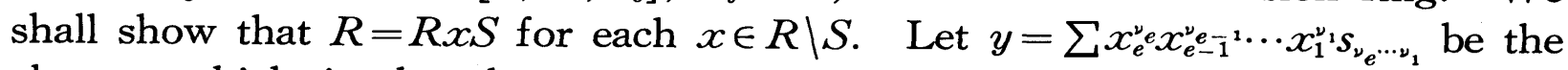
element which is the shortest non zero relation contained in $R x S$. Then, without loss of generality, we may assume that $y \in R \mid S$. Since $T$ is $T_{z} S_{r^{-}}$ irreducible and each $x_{i}$ is regular, we may assume that the constant term of $y$ is 1 . Thus $s y-y s=0$, thst is, $R=R y S=R x S$. The converse is clear.

In what follow, we assume that $T$ is a $q$-dimensional abelian extension over $S$ with respect to $\mathfrak{S}=\left(\tau_{1}\right) \times\left(\tau_{2}\right) \times \cdots \times\left(\tau_{m}\right)$, \# $\tau_{i}=q_{i}$ such that $T$ is $T_{z} S_{r^{-}}$ irreducible and $V_{T}(T) \ni \zeta$. 
Theorem 4.2. In order that $T$ can be regularly embedded in a qndimensional abelian extension $R / S$ such that $R / T$ is an $n$-dimensional Kummer extension such that $V$ is a division ring, it is necessary and sufficient that there exist automorphisms $\rho_{1}, \rho_{2}, \cdots, \rho_{m+k}(k>-m)$ in $T,\left(s_{i j}\right)$ an $(m+k) \times(m+k)$ matrix with entries in $T^{\bullet},\left(t_{i}\right)$ a $1 \times(m+k)$ matrix with entries in $T^{\bullet}$ satisfying the conditions (1)-(5) of Theorem 4.1 (in $T)$ and $\left(b_{i j}\right)$ an $(m+k) \times(m+k)$ matrix with entries in $T^{\bullet}$ satisfying

(1) $\tau_{j}^{-1} \rho_{i}^{-1} \tau_{j} \rho_{i}=\tilde{b}_{i j}$

(3) $L N_{n_{i}}\left(b_{i j} ; \rho_{i}\right)=t_{i}^{-1} \cdot t_{i} \rho_{j}$,

(4) $s_{i j}\left(b_{i k} \rho_{j}\right) b_{j k}=\left(b_{j k} \rho_{i}\right) b_{i k}\left(s_{i j} \tau_{k}\right)$,

(5) $b_{i k}\left(b_{i j} \tau_{k}\right)=b_{i j}\left(b_{i k} \tau_{j}\right)$

(6) If $g(X)$ is a polynomial in $T\left[\mathfrak{X}_{m+k} ; \mathfrak{B}_{m+k}\right] \backslash M, M=\left(X_{1}^{n_{1}}-t_{1}, X_{2}^{n_{2}}-\right.$ $\left.t_{2}, \cdots, X_{m+k}^{n_{m+k}}-t_{m+k}\right)$, then $\sum_{i} f_{i}(X) g(X) s_{i}=1$ modulo $M$ for some $f_{i}(X) \in T$ $\left[\mathfrak{X}_{m+k} ; \mathfrak{P}_{m+k}\right], s \in S$.

More precisely, if there exists $\left\{\rho_{1}, \rho_{2}, \cdots, \rho_{e},\left(s_{i j}\right),\left(t_{i}\right),\left(b_{i j}\right)\right\}$ satisfying the conditions, then $M=\left(X_{1}^{n_{1}}-t_{1}, \cdots, X_{m+k}^{n_{m_{k}}}-t_{m+k}\right) T\left[\mathfrak{X}_{m+k} ; \mathfrak{P}_{m+k}\right]$ and $R^{* *}=T\left[y_{1}\right.$, $\left.\cdots, y_{m}, y_{m+1}, \cdots, y_{m+k}\right] \cong T\left[\mathfrak{X}_{m+k} ; \mathfrak{\beta}_{m+k}\right] / M, y_{i}$ is the residue class of $X_{i}$ modulo $M$, is a requested one with generating automorphisms $\sigma_{1}^{*}, \sigma_{2}^{*}, \cdots, \sigma_{m+k}^{*}$ such that $\left(y_{i} t\right) \sigma_{j}^{*}=y_{i} b_{i j} t \tau_{j}(t \in T)$ and $\mathbb{S}^{*} \mid T=\mathfrak{S}$ where $\mathbb{S}^{*}=\left(\sigma_{1}^{*}\right) \times\left(\sigma_{2}^{*}\right) \times \cdots \times\left(\sigma_{m}^{*}\right) \times$ $\left(\sigma_{m+1}^{*}\right) \times \cdots \times\left(\sigma_{m+k}^{*}\right)$ if $k>0$ and $\mathbb{S}^{*}=\left(\sigma_{1}^{*}\right) \times\left(\sigma_{2}^{*}\right) \times \cdots \times\left(\sigma_{m+k}^{*}\right) \times\left(\tau_{m+k+1}\right) \times \cdots \times\left(\tau_{m}\right)$ if $k<0$. Conversely, if $T / S$ be regularly embedded in a qn-dimensional abelian extension with respect to $\mathbb{B}=\left(\sigma_{1}\right) \times\left(\sigma_{2}\right) \times \cdots \times\left(\sigma_{m+k}\right)$ such that $R / T$ is an $n$-dimensional Kummer extension, then there exists $\left\{\rho_{1}, \rho_{2}, \cdots, \rho_{e},\left(s_{i j}\right),\left(t_{i}\right)\right.$, $\left.\left(b_{i j}\right)\right\}$ satisfying the conditions in Theorem 4.1 (in $T$ ) and (1)-(5) that there holds a $T$-isomorphism $\varphi^{*}: R^{*} \cong R$ with $\varphi^{*} \sigma_{i}=\sigma_{i}^{*} \varphi^{*}$ for each $i=1,2, \cdots, m+k$.

Proof. Firstly we set $b_{i j} \begin{cases}=\zeta^{n / n_{i}} & \text { if } k>0 \text { and } i=j>m \\ =1 & \text { if } k>0, i \neq j \text { and } i, j>m\end{cases}$ or $k<0$ and $j>m+k$

Then we can easily checked that they satisfy the conditions (1)-(5).

Let $\Psi_{j}(j=1,2, \cdots, m+k)$ be the mappings of $T\left[\mathfrak{X}_{m+k} ; \mathfrak{P}_{m+k}\right]$ defined by $\sum X_{m+k}^{\nu_{m+k}} \cdots X_{1}^{\nu_{1}} t_{\nu_{m+k} \cdots \nu_{1}} \rightarrow \sum\left(X_{m+k} b_{m+k j}\right)^{\nu_{m+k}} \cdots\left(X_{1} b_{1 j}\right)^{\nu_{1}}\left(t_{\nu_{m+k} \cdots \nu_{1}} \tau_{j}\right)$ (we set $\tau_{j}=1$ if $j>m)$. Then we can prove that $\Psi_{j}$ is an automorphism by induction on the number of indeterminates.

$\left(t \Psi_{j}\right) X_{i} \Psi_{j}=\left(t \tau_{j}\right)\left(X_{i} b_{i j}\right)=X_{i} t \tau_{i} \rho_{i} b_{i j}$ and $\left(X_{i} t \rho_{i}\right) \Psi_{j}=X_{i} b_{i j}+t \rho_{i} \tau_{j}$ and hence the condition (1) yields $\left(t X_{i}\right) \Psi_{j}=\left(X_{i} t \rho_{i}\right) \Psi_{j}$, and further, $\# \Psi_{j}=n_{j} q_{j}$ (by (2)) shows that $\Psi_{j}$ is an automorphism in $T\left[X_{i} ; \rho_{i}\right]$. Hence we assume that the assertion is true for each $T\left[\mathfrak{X}_{m+k-1} ; \mathfrak{P}_{m+k-1}\right]$. Then it suffices to prove 
$\left(Y \Psi_{j}\right)^{\cdot}\left(X_{m+k} \Psi_{j}\right)=\left(X_{m+k} \Psi_{j}\right)\left(Y P_{m+k} \Psi_{j}\right)$ where $Y \in T\left[\mathfrak{X}_{m+k-1} ; \mathfrak{P}_{m+k-1}\right]$. Since $\left(Y \Psi_{j}\right)\left(X_{m+k} \Psi_{j}\right)=Y \Psi_{j}\left(X_{m+k} b_{m+k j}\right)=X_{u+k}\left(Y \Psi_{j} P_{m+k}\right) b_{m+k j}$ and $\left(X_{m+k} \Psi_{j}\right) Y P_{m+k} \Psi_{j}$ $=X_{m+k} b_{m+k j}\left(Y P_{m+k} \Psi_{j}\right)$, it suffices to prove $Y \Psi_{j} P_{m+k}=Y P_{m+k} \Psi_{j} \widetilde{b}_{m+k j}$. Now, $X_{i} \Psi_{j} P_{m+k}=\left(X_{i} b_{i j}\right) P_{m+k}=X_{i} s_{i m+k} b_{i j} \rho_{m+k}$ and $X_{i} P_{m+k} \Psi_{j} \widetilde{b}_{m+k j}=b_{m+k j} X_{i} b_{i j}\left(s_{i m+k} \tau_{j}\right)$ $b_{m+k j}^{-1}=X_{i} b_{m+k j} \rho_{i} b_{i j}\left(s_{i m+k} \tau_{j}\right) b_{m+k j}^{-1}$. Hence the condition (4) shows that $X_{i} \Psi_{j} P_{m+k}$ $=X_{i} P_{m+k} \Psi_{j} \widetilde{b}_{m+k j}$. Thus we assume that $Y \Psi_{j} P_{m+k}=Y P_{m+k} \Psi_{j} \widetilde{b}_{m+k j}$ where $Y=$

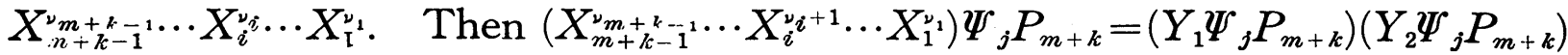
$=Y_{1} P_{m+k} \Psi_{j} \widetilde{b}_{m+k j} Y_{2} P_{m+k} \Psi_{j} \widetilde{b}_{m+k j}=\left(X_{m+k-1}^{\nu} \cdots X_{i}^{\nu} i^{+1} X_{1}^{\nu_{1}}\right) P_{m+k} \Psi_{j} \widetilde{b}_{m+k j}$ where $Y_{1}=$ $X_{m+k-1}^{\nu m+k-1} \cdots X_{i}^{\nu} i$ and $Y_{2}=X_{i} \cdots X_{1}^{\nu}$. Consequently, we have $Y \Psi_{j} P_{m+k}=$ $Y P_{m+k} \Psi \widetilde{b}_{m+k j}$ for each $Y \in T\left[\mathfrak{X}_{m+k-1} ; \mathfrak{P}_{m+k-1}\right]$.

$\left(X_{i}^{n_{i}} t_{i}^{-1}-1\right) \Psi_{j}=X_{i}^{n_{i}} L N_{n_{i}}\left(b_{i j} ; \rho_{i}\right) t_{i}^{-1} \tau_{j}-1$ and the condition (3) show that $M \Psi_{j}=M$. Hence each $\Psi_{j}$ induces an automorphism $\sigma_{j}^{*}$ in $R^{*}=T\left[y_{1}, \cdots\right.$, $\left.y_{m+k}\right] \cong T\left[\mathfrak{X}_{m+k} ; \mathfrak{\beta}_{m+k}\right] / M, y_{i}$ is the residue class of $X_{i}$ modulo $M$, is a $q n$ dimensional simple ring extension over $S$. Moreover, by (2), $\# \sigma_{j}^{*}\left\{\begin{array}{ll}=q_{j} n_{j} & \text { if } j \leqq m \\ =n_{j} & \text { if } j>m\end{array}\right.$.

$y_{i} \sigma_{j}^{*} \sigma_{k}^{*}=y_{i} b_{i k}\left(b_{i j} \tau_{k}\right)=y_{i} b_{i j}\left(b_{i k} \tau_{j}\right)=y_{i} \sigma_{k}^{*} \sigma_{j}^{*}$ by condition (5) shows that $\sigma_{j}^{*} \sigma_{k}^{*}$ $=\sigma_{k}^{*} \sigma_{j}^{*}$. Let $\sigma^{*} \in\left(\sigma_{i}^{*}\right)_{\cap}\left(\sigma_{1}^{*}, \sigma_{2}^{*}, \cdots, \sigma_{i-1}^{*}\right)$. Then $\sigma^{*}=\sigma_{i}^{* \alpha_{i}}=\sigma_{1}^{* \alpha_{1}} \sigma_{2}^{* \alpha_{2}} \cdots \sigma_{i-1}^{* \alpha_{i-1}}$ and $\sigma^{*} \mid T=\tau_{i}^{\alpha} i=\tau_{1}^{\alpha_{1}} \cdots \tau_{i-1}^{\alpha}{ }_{i-1}$ show that each $\alpha_{j}$ is a mutiple of $q_{j}$, and hence $y_{i} \sigma^{*}=$ $y_{i} \sigma_{1}^{* \alpha_{1}} \sigma_{2}^{* \alpha_{2}} \cdots \sigma_{i-1}^{* \alpha_{i-1}}=y_{i}$ by $R N_{q_{j}}\left(b_{i j} ; \tau_{j}\right)=1$. Consequently, we have $\sigma^{*}=1$. Thus, (S), the group generated by $\sigma_{1}^{*}, \sigma_{2}^{*}, \cdots, \sigma_{m+k}^{*}$, is a direct product of each $\sigma_{i}^{*}$, and $J\left(S^{*}, R^{*}\right)=J\left(S^{*} \mid T, T\right)=J(\mathfrak{S}, T)=S$.

Finally, if (6) is satisfied, then by Lemma $4.1, V^{*}$ is a division ring.

Conversely, let $T / S$ can be regularly embedded in a $q n$-dimensional abelian extension $R / S$ with respect to $\mathbb{S}=\left(\sigma_{1}\right) \times\left(\sigma_{2}\right) \times \cdots \times\left(\sigma_{m}\right) \times \cdots \times\left(\sigma_{m+k}\right)$ if $k \geqq 0$ and SS $=\left(\sigma_{1}\right) \times \cdots \times\left(\sigma_{m+k}\right) \times\left(\tau_{m+k+1}\right) \times \cdots \times\left(\tau_{m}\right)$ if $-m<k<0$ such that $\# \sigma_{j}=\left(\# \tau_{j}\right) n_{j}$, $\sigma_{j} \mid T=\tau_{j}$ if $j \leqq m$ and $\# \sigma_{j}=n_{j}, \sigma_{j} \mid T=1$ if $j>m$.

Since $V$ is a field and $[R: T]=n, R / T$ is an $n$-dimensional Kummer extension with respect to $\mathcal{S S}(T)=\left(\sigma_{1}^{q_{1}}\right) \times\left(\sigma_{2}^{q_{2}}\right) \times \cdots \times\left(\sigma_{m}^{q_{m}}\right) \times\left(\sigma_{m+1}\right) \times \cdots \times\left(\sigma_{m+k}\right)$. Then

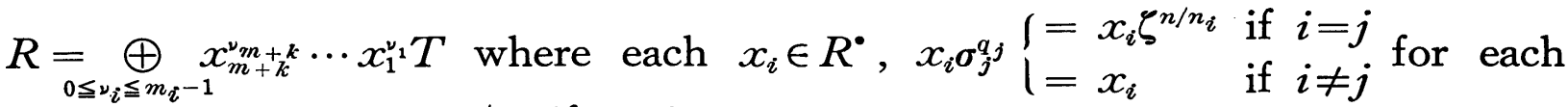
$j \leqq m$ and $x_{i} \sigma_{j}\left\{\begin{array}{ll}=x_{i} \zeta^{n / n_{i}} & \text { if } i=j \\ =x_{i} & \text { if } i \neq j\end{array}\right.$ for each $j>m . \quad$ Then $\rho_{i}=\tilde{x}_{i}^{-1} \mid T, t_{i}=x_{i}^{n_{i}}$, $s_{i j}=x_{i}^{-1} x_{j}^{-1} x_{i} x_{j}$ satisfy the conditions (1)-(5) of Theorem 4.1 (in $T$ ).

Now we set $b_{i j}=x_{i}^{-1}\left(x_{i} \sigma_{j}\right)$. Then $b_{i j} \sigma_{k}^{q_{k}}=x_{i}^{-1} \sigma_{k}^{q_{k}} x_{i} \sigma_{j} \sigma_{k}^{q_{k}}=$ $\left\{\begin{array}{ll}=x_{i}^{-1} \zeta^{-n / n_{i}}\left(x_{i} \sigma_{j} \zeta^{n / n_{i}}\right)=x_{i}^{-1} x_{i} \sigma_{j}=b_{i j} & \text { if } i=j \\ =x_{i}^{-1}\left(x_{i} \sigma_{j}\right)=b_{i j} & \text { if } i \neq j\end{array}\right.$ for each $k \leqq m$ and $b_{i j} \sigma_{k}=\left(x_{i}^{-1}\right.$ $\left.\left(x_{i} \sigma_{j}\right)\right) \sigma_{k}=b_{i j}$ if $k>m$ show that $b_{i j} \in T$.

Next, $t b_{i j}=x_{i}^{-1}\left(x_{i} \sigma_{j}\right) t\left(x_{i}^{-1} \sigma_{j}\right) x_{i}=x_{i}^{-1}\left(x_{i} t \tau_{j}^{-1} x_{i}^{-1}\right) \sigma_{j} x_{i}=x_{i}^{-1}\left(t \tau_{j}^{-1} \rho_{i}^{-1}\right) \cdot \sigma_{j} x_{i}=$ 
$x_{i}^{-1}\left(t \tau_{j}^{-1} \rho_{i}^{-1} \tau_{j}\right) x_{i}=t \tau_{j}^{-1} \rho_{i}^{-1} \tau_{j} \rho_{i}$.

$R N_{q_{j}}\left(b_{i j} ; \tau_{j}\right)=x_{i}^{-1}\left(x_{i} \sigma_{j}\right)\left(x_{i}^{-1} \sigma_{j}\right)\left(x_{i} \sigma_{j}^{2}\right) \cdots\left(x_{i}^{-1} \sigma_{j}^{q_{j}-1}\right)\left(x_{i} \sigma_{j}^{a_{j}}\right)=x_{i}^{-1} x_{i} \sigma_{j}^{q_{j}}$

$\left\{\begin{array}{ll}=\zeta^{n / n_{i}} & \text { if } i=j \\ =1 & \text { if } i \neq j\end{array}\right.$ by the acts of $\sigma_{j}^{q_{j}}$ on $x_{i}$.

$L N_{n_{i}}\left(b_{i j} ; \rho_{i}\right)=\left(x_{i}^{1-n_{i}} x_{i}^{-1}\left(x_{i} \sigma_{j}\right) x_{i}^{n_{i}-1}\right)\left(x_{i}^{2-n_{i}} x_{i}^{-1}\left(x_{i}^{-1} \sigma_{j}\right) x_{i}^{n_{i}-2}\right) \cdots x_{i}^{-1}\left(x_{i} \sigma_{j}\right)=x_{i}^{-n_{i}}$ $\left(x_{i} \sigma_{j}\right)^{n_{i}}=x_{i}^{-n_{i}}\left(x_{i}^{n_{i}} \sigma_{j}\right)=t_{i}^{-1}\left(t_{i} \sigma_{j}\right)$.

$s_{i j}\left(b_{i k} \rho_{j}\right) b_{j k}=\left(x_{i}^{-1} x_{j}^{-1} x_{i} x_{j}\right)\left(x_{j}^{-1} x_{i}^{-1}\left(x_{i} \sigma_{k}\right) x_{j}\right) x_{j}^{-1}\left(x_{j} \sigma_{k}\right)=x_{i}^{-1} x_{j}^{-1}\left(x_{i} \sigma_{k}\right)\left(x_{j} \sigma_{k}\right)=$ $\left(x_{i}^{-1}\left(x_{j}^{-1} x_{j} \sigma_{k}\right) x_{i}\right)\left(x_{i}^{-1}\left(x_{j}^{-1} \sigma_{k}\right)\left(x_{i} \sigma_{k}\right)\left(x_{i} \sigma_{k}\right)\right)=b_{j k} \rho_{i}\left(x_{i}^{-1}\left(x_{i} \sigma_{k}\right)\left(x_{i}^{-1} \sigma_{k}\right)\left(x_{j}^{-1} x_{i} x_{j}\right) \sigma^{k}\right)=$ $b_{j k} \rho_{i} b_{i k}\left(s_{i j} \tau_{k}\right)$.

$x_{i}\left(b_{i k}\left(b_{i j} \tau_{k}\right)\right)=x_{i} \sigma_{j} \sigma_{k}=x_{i} \sigma_{k} \sigma_{j}=x_{i} b_{i j}\left(b_{i k} \tau_{j}\right)$ shows that $b_{i k}\left(b_{i j} \tau_{k}\right)=b_{i j}\left(b_{i k} \tau_{j}\right)$.

Finally, since $V$ is a division ring, $T_{t} S_{r}$-irreducibility of $T$ yields at once the condition (6) by Lemma 4.1 in $T\left[\mathfrak{X}_{m+k} ; \mathfrak{P}_{m+k}\right]$ where $X_{i} P_{j}=X_{i} s_{i j}$ and $S \mid P_{j}=\rho_{j}$.

The condition (6) of Theorem 4.2 was need only to see that the centralizer $V^{*}$ is a division ring. Accordingly, we readily obtain the following

Corollary 4. 5. Let $S$ be a division ring, and let $T / S$ be a q-dimensional abelian division ring extension satisfying the condition of Theorem 4.2. In order that $T / S$ can be regularly embedded in a qn-dimensional abelian division ring extension $R / S$ such that $R / T$ is an n-dimensional Kummer extension, it is necessary and sufficient that there exist $\left\{\rho_{1}, \rho_{2}, \cdots, \rho_{e},\left(s_{i j}\right),\left(t_{i}\right)\right.$, $\left.\left(b_{i j}\right)\right\}$ satisfying $(1)-\left(5^{\prime}\right)$ of Corollary $4.3($ in $T)$ and $(1)-(5)$ of Theorem 4.1.

\section{References}

[1] S. A. Amitsur: Non-commutative cyclic fields, Duke Math. J., Vol. 21 (1954), 87-105.

[2] P. M. CoHN: On a class of binomial extensions, Illinois J. of Math., Vol. 10 (1966), 418-424.

[3] K. Kishimoto: On cyclic extensions of simple rings, J. Fac Sci. Hokkaido Univ., Vol. 19 (1966), 74-85.

[4] - On polynomial extensions of simple rings, J. Fac. Sci. Hokkaido Univ., Vol 19 (1966), 169-180,

[5] K. Kishimoto, T. OnODERA and H. Tominaga : On normal basis theorem and the extension dimension, J. Fac. Sci. Hokkaido Univ., Vol. 18 (1964), 82-88.

[6] T. Nagahara, T. OnOdera and H. Tominaga: On normal basis theorem and strictly Galois extensions, Math. J. Okayama Univ., Vol. 8 (1958), 133-142.

[7] T. NAGAhara and H. TOMinaga: On Galois and locally Galois extensions of simple rings, Math. J. Okayama Univ., Vol. 10 (1961), 143-166.

[ 8 ] N. Nobusawa and H. Tominaga: Some remarks on strictly Galois extensions of simple rings, Math. J. Okayama Univ., Vol. 9 (1959), 13-17.

Department of Mathematics Shinshû University, Matsumoto

(Received May 20, 1967) 